\title{
Three Small-Receptive-Field Ganglion Cells in the Mouse Retina Are Distinctly Tuned to Size, Speed, and Object Motion
}

\author{
기 Jason Jacoby ${ }^{1}$ and ${ }^{-G}$ Gregory W. Schwartz ${ }^{1,2}$ \\ 'Department of Ophthalmology, 2Department of Physiology, Feinberg School of Medicine, Northwestern University, Chicago, Illinois 60611
}

Retinal ganglion cells (RGCs) are frequently divided into functional types by their ability to extract and relay specific features from a visual scene, such as the capacity to discern local or global motion, direction of motion, stimulus orientation, contrast or uniformity, or the presence of large or small objects. Here we introduce three previously uncharacterized, nondirection-selective ON-OFF RGC types that represent a distinct set of feature detectors in the mouse retina. The three high-definition (HD) RGCs possess small receptive-field centers and strong surround suppression. They respond selectively to objects of specific sizes, speeds, and types of motion. We present comprehensive morphological characterization of the HD RGCs and physiological recordings of their light responses, receptive-field size and structure, and synaptic mechanisms of surround suppression. We also explore the similarities and differences between the HD RGCs and a well characterized RGC with a comparably small receptive field, the local edge detector, in response to moving objects and textures. We model populations of each RGC type to study how they differ in their performance tracking a moving object. These results, besides introducing three new RGC types that together constitute a substantial fraction of mouse RGCs, provide insights into the role of different circuits in shaping RGC receptive fields and establish a foundation for continued study of the mechanisms of surround suppression and the neural basis of motion detection.

Key words: feature selectivity; object motion; retina; retinal ganglion cell

\section{Significance Statement}

The output cells of the retina, retinal ganglion cells (RGCs), are a diverse group of $\sim 40$ distinct neuron types that are often assigned "feature detection" profiles based on the specific aspects of the visual scene to which they respond. Here we describe, for the first time, morphological and physiological characterization of three new RGC types in the mouse retina, substantially augmenting our understanding of feature selectivity. Experiments and modeling show that while these three "high-definition" RGCs share certain receptive-field properties, they also have distinct tuning to the size, speed, and type of motion on the retina, enabling them to occupy different niches in stimulus space.

\section{Introduction}

Distinct aspects of a visual scene are relayed to higher brain regions by trains of action potentials originating from $>30$ types of

Received Sept. 6, 2016; revised Nov. 14, 2016; accepted Nov. 25, 2016.

Author contributions: J.J. and G.W.S. designed research; J.J. and G.W.S. performed research; J.J. and G.W.S. analyzed data; J.J. and G.W.S. wrote the paper.

This work was supported by the Ruth L. Kirschstein National Research Service Award Postdoctoral Fellowship 1F32EY025930-01, National Institutes of Health Grant DP2-DEY026770A, and a Research to Prevent Blindness Career Development Award. We thank our laboratory manager, Susan Wohlgenant, for her extensive experimental preparation and technical assistance. We thank Sam Cooler, for helping to produce an effective and important centering stimulus for the HD RGCs, and Dr. Adam Mani, for various data analysis codes used in this research study. We thank Jianhua Cang, Tiffany Schmidt, Steven H. DeVries, and William Grimes for their commentary on early drafts of the manuscript.

The authors declare no competing financial interests.

Correspondence should be addressed to Dr. Gregory Schwartz, Tarry 5-733, 300 East Superior Street, Chicago, IL 60611. E-mail: greg.schwartz@northwestern.edu. retinal ganglion cells (RGCs; Masland, 2012b; Baden et al., 2016). In 1959, Lettvin and colleagues first described the net convexity detector in the frog retina, an RGC exclusively activated by small objects of equal or lesser size than the receptive-field (RF) center. Spiking output of this cell was abolished entirely when the object and background were moved together in synchrony, but activated when the object was moved against a stationary background; they dubbed this RGC type a "bug perceiver" (Lettvin et al., 1959). Nearly a decade later, a functional homolog was reported in the rabbit retina by W.R. Levick. The local edge detector (LED) RGC was also optimally excited when the RF center was precisely stimulated; visual stimuli that concurrently fell upon 
the RF surround resulted in strong attenuation or full suppression of the LED RGC's spiking output (Levick, 1967). More recently, a similar cell in the mouse retina was found to also exhibit a compact dendritic field and strong surround suppression (Zhang et al., 2012). While LED RGCs in mice indeed incorporate the small RF and strong surround suppression vital to selectively detect small objects, several groups have assigned the LED RGC an additional functional role: object-motion sensitivity (Olveczky et al., 2003; Zhang et al., 2012; Kim et al., 2015).

Is the LED RGC the sole small-object detector of the mouse retina, or are other types of nondirection-selective (non-DS) mouse RGCs with small RF centers involved in representing small stationary or moving objects? Large-scale morphological studies suggest that such RGC types may exist. Small dendritic field RGCs are best represented in the published mouse electron microscopic (EM) connectome (Helmstaedter et al., 2013). Based on morphological differences and mosaic spacing, the EM study identified $\geq 3$ other RGC types with comparable density to the putative LED (W3b) RGC which stratify at the same level, at the ON-OFF interface of the inner plexiform layer (IPL). A larger online database of RGC morphologies has also identified $\geq 3$ high-density RGC types costratified with the putative LED RGC [Eyewire Cell Museum; http://museum.eyewire.org, including the most numerous RGC in that dataset (personal communication, Sebastian Seung)]. Characterizing these RGC types is important because (1) their high density implies that they represent a substantial fraction of the total RGC population, constituting a major step in the stated goal of identifying all the mouse RGC types (Sanes and Masland, 2015), and (2) they may reveal important principles about the way small stationary and moving stimuli are encoded in the retina.

Here we show the reliable physiological targeting of three, non-DS ON-OFF RGC types in wild-type mouse retina that we name the "high-definition" (HD) family of RGCs [HD1, HD2, and UHD (ultra-HD)]. Similar to the LED RGC, HD RGCs are activated by small spots of positive or negative contrast and exhibit strong surround suppression. We provide several lines of evidence that these three HD RGC types and the LED RGC are in fact distinct RGC types. This evidence includes morphological analysis with putative assignments to types from the EM datasets and response profiles under a variety of visual stimulus conditions. We also reveal that the synaptic mechanisms of surround suppression in each RGC type are distinct, and explore their sensitivity to a variety of motion stimuli. Together, these results provide the first morphological and physiological characterization, to our knowledge, of three RGC types in the mouse retina, and they offer new insights into the varied mechanisms of surround suppression and the retinal representation of object motion.

\section{Materials and Methods}

Recording. Wild-type mice of either sex (4-10 weeks old) were darkadapted overnight. Dissection and excision of retinal tissue was performed under infrared illumination $(900 \mathrm{~nm})$ using night-vision goggles and night-vision dissecting scope monocular attachments. Research animals were killed in accordance with all animal care standards provided by Northwestern University. A piece of retina was placed onto a glass coverslip coated with poly-D-lysine, ganglion cell layer facing upward for easy recording access. Tissue was superfused with carbogenated Ames solution warmed to $32^{\circ} \mathrm{C}$. The identity of RGCs was confirmed with cell-attached capacitive spike trains in response to various light stimuli. Voltage-clamp recordings were performed with cesium-based intracellular solution. For more detailed description of the solutions, equipment, and protocols used, refer to Jacoby et al., 2015 or Nath and Schwartz, 2016.
Visual stimuli. Visual stimuli were presented with a custom-designed light-projection device capable of controlling patterned visual stimulation at high frame rates (1.4 kHZ). Stimuli were generated on a $912 \times$ 1140-pixel digital projector using the blue ( $450 \mathrm{~nm}$ ) LED and focused on the photoreceptors. We report light intensities in rod isomerizations $\left(\mathrm{R}^{*}\right)$ per rod per second. Based on the spectrum of our blue LED, the spectral sensitivities of mouse opsins, and collecting areas of mouse photoreceptors, each $\mathrm{R}^{\star}$ corresponds to 0.3 isomerizations per $\mathrm{M}$-cone opsin and $6 \times 10^{-4}$ isomerizations per S-cone opsin.

Stimuli were first aligned to the RF center of each cell using a series of $25 \mu \mathrm{m}$ (diameter) spots to map the RF. All following stimuli were presented in pseudorandom sequence, and stimuli from darkness were presented at $200 \mathrm{rod} \mathrm{R}^{\star} / \mathrm{rod} / \mathrm{s}$. Spatial and temporal tuning curves were generated by moving light bars $\left(200 \mathrm{R}^{\star} / \mathrm{rod} / \mathrm{s}\right)$ from darkness through the RF center that differed in width $(100,200,400 \mu \mathrm{m})$ and speed $(500,1000$, $2000,4000,8000 \mu \mathrm{m} / \mathrm{s}$ ). All moving bars presented were $1000 \mu \mathrm{m}$ long to quantify $\mathrm{ON}$ and $\mathrm{OFF}$ responses independently. Flashed texture stimuli $(0.4,11,26,55$, and $110 \mu \mathrm{m}$ texture scales) were randomly presented as full field $(3000 \times 3000 \mu \mathrm{m})$ or, in a separate experiment, as randomly presented textures solely onto the RF center (diameter dependent upon on specific HD cell type RF center). Each texture scale contained the same amount of positive and negative contrast, and textures were presented from a mean background light level of $1000 \mathrm{R}^{\star} / \mathrm{rod} / \mathrm{s}$. Stimuli that probed object-motion sensitivity used the same texture profiles mentioned above, but differentially or concurrently moved these stimuli to probe responses to center, surround, global, or differential motion (mean background light level of $\left.500 \mathrm{R}^{\star} / \mathrm{rod} / \mathrm{s}\right)$. All other light levels used are presented in the text in $\mathrm{R}^{\star} / \mathrm{rod} / \mathrm{s}$.

Analysis. All data in the paper are reported as mean \pm SEM. We make comparisons for statistical significance between conditions and between cell types with Student's paired one-sided $t$ tests or the KolmogorovSmirnov test. Spike-counting windows include the full duration of stimulus presentation or, for moving bars, the full time period over which the bar was on the screen. Latency of spike responses are defined by the time the spike rate crosses its half-maximal value. Direction selectivity index (see Fig. 2) was calculated using the vector-sum method (Trenholm et al., 2011). Spatial and temporal tuning curves used peak firing rate (in hertz) to determine response properties, and were split independently for the leading $(\mathrm{ON})$ and trailing edge (OFF) of a bright bar. Texture matrix stimuli were quantified by counting the total number of spikes from stimulus (full field or RF center) onset through $500 \mathrm{~ms}$ after the stimulus offset to incorporate both ON and OFF responses to a specific flashed texture. Data were analyzed with a custom open-source Matlab analysis package (github.com/SchwartzNU/SymphonyAnalysis), and figures were assembled in Igor Pro or Adobe Illustrator.

Imaging. For dendritic stratification and morphological imaging, target cells were injected through patch pipettes with Neurobiotin tracer and labeled with streptavidin. Fixed tissues were imaged on a laser scanning confocal microscope through an inverted $60 \times$ oil-immersion objective and scanned with GaAsP detectors. Streptavadin-conjugated and ChAT-conjugated fluorescent labeling was stimulated at 488 and $640 \mathrm{~nm}$, respectively. All confocal images were acquired in $0.15-0.2 \mu \mathrm{m}$ steps in the $z$-axis. Confocal imaging was performed at the Nikon Imaging Center at Northwestern University's Feinberg School of Medicine using Nikon Elements software. Images were analyzed using ImageJ/Fiji. Dendrites were traced and computationally flattened relative to the ChAT bands using the algorithm developed by Sümbül et al. (2014). For a more detailed description of staining and imaging protocols, refer to Jacoby et al., 2015 or Nath and Schwartz, 2016.

Object-tracking model. The model for tracking an object (see Fig. 10) was constructed in Matlab based on measured parameters for HD and LED RGCs. The stimulus was a square of 100,200 , or $400 \mu \mathrm{m}^{2}$ moving in a quasirandom walk for $2 \mathrm{~s}$ inside a 1-mm-square arena. Time was sampled in $10 \mathrm{~ms}$ bins, and in each time bin, a velocity was chosen from the distribution in Figure $10 \mathrm{~A}$, and a direction of movement was chosen from a uniform random distribution. To add continuity to the trajectory, the motion-direction vector was smoothed with an $80 \mathrm{~ms}$ sliding average filter. 
Cell mosaics were constructed as noisy hexagonal grids as previously described (Schwartz et al., 2012) with an SD of $10 \mu \mathrm{m}$ for the cell-position noise. The average spacing between cells in the mosaic was $70 \mu \mathrm{m}$ for HD1, HD2, and LED RGCS, and $50 \mu \mathrm{m}$ for UHD RGCs. These values were chosen as half the dendritic diameter (Table 1) rounded to the nearest $10 \mu \mathrm{m}$, consistent with previous estimates of the dendritic coverage factor of RGCs (Peichl and Wässle, 1983; Yang and Masland, 1994; Bleckert et al., 2014). ON $\alpha$ and ON-OFF DS RGCs were spaced at 140 and $100 \mu \mathrm{m}$, respectively, according to previous reports of their dendritic diameters. Mosaics extended to 2 $\mathrm{mm}$ squares even though the stimulus occupied only to the central 1 $\mathrm{mm}$ to avoid edge effects.

RFs for each cell in the mosaic were modeled as two-dimensional difference of Gaussians fit from the response profiles in Figure 2 (and a similar profile, not shown for ON $\alpha$ RGCs). The fits included three parameters: the SD of the center Gaussian $\left(\sigma_{\mathrm{C}}\right)$, the $\mathrm{SD}$ of the surround $\left(\sigma_{\mathrm{S}}\right)$, and the surround/center ratio $\left(R_{\mathrm{SC}}\right)$. Values for each cell type were as follows: HD1: $\sigma_{\mathrm{C}}, 28 \mu \mathrm{m} ; \sigma_{\mathrm{S}}, 50 \mu \mathrm{m} ; R_{\mathrm{SC}}, 0.99 ; \mathrm{HD} 2: \sigma_{\mathrm{C}}, 52 \mu \mathrm{m} ; \sigma_{\mathrm{S}}$, $125 \mu \mathrm{m} ; R_{\mathrm{SC}}, 0.84$; UHD: $\sigma_{\mathrm{C}}, 20 \mu \mathrm{m} ; \sigma_{\mathrm{S}}, 56 \mu \mathrm{m} ; R_{\mathrm{SC}}, 1$; LED: $\sigma_{\mathrm{C}}, 34 \mu \mathrm{m}$; $\sigma_{\mathrm{S}}, 72 \mu \mathrm{m} ; R_{\mathrm{SC}}, 1$; ON-OFF DS: $\sigma_{\mathrm{C}}, 32 \mu \mathrm{m} ; \sigma_{\mathrm{S}}, 102 \mu \mathrm{m} ; R_{\mathrm{SC}}, 0.77$; ON $\alpha$ : $\sigma_{\mathrm{C}}, 70 \mu \mathrm{m} ; \sigma_{\mathrm{S}}, 231 \mu \mathrm{m} ; R_{\mathrm{SC}}, 0.41$. At each time step, the RF overlap of each cell in the mosaic with the stimulus was computed with a spatial dot product to yield the RF activation of each cell (see Fig. 10B). The local velocity of the stimulus was computed as the average velocity over the preceding $40 \mathrm{~ms}$, and each nonzero RF activation value was multiplied by a peak firing rate value interpolated from the speed and width tuning curves for each cell type (see Fig. 10C) presented in Figure 8. Noise models for each cell type were constructed by measuring the SD across trials of the spike count within $100 \mathrm{~ms}$ centered on the time of the peak firing-rate response. For each cell and each condition, we plotted the SD against the peak firing rate and fit the resulting relationship as a linear relationship (see Fig. 10D). The noise model predicted an SD of noise that was added to each model RGC in the mosaic based on its firing rate on each time step. Values of the noise model fits for each cell type were as follows, listed as slope, intercept: HD1: $-0.0085,20 \mathrm{~Hz}$; HD2: 0, $16 \mathrm{~Hz}$; UHD: 0.027, $13 \mathrm{~Hz}$; LED: 0.073, 6.8 Hz; ON-OFF DS: 0.039, $5.1 \mathrm{~Hz}$; ON $\alpha: 0.021,15 \mathrm{~Hz}$. The resulting firing rates with added noise were rectified to non-negative values (see Fig. 10E).

We computed the neural estimate of the stimulus location on each frame as the center of mass of the population firing response. Tracking error was calculated as the average displacement between the actual stimulus location and this neural estimate.

\section{Results \\ Morphological and physiological characterization of HD RGCs}

As part of a large-scale survey of the light responses of mouse RGCs, we located the RF center of each cell using a series of briefly presented, small spots (see Materials and Methods). Following determination of the RF center, we probed RGC spike responses with a $200-\mu \mathrm{m}$-diameter spot of light from darkness (200 rod isomerizations $\mathrm{R}^{\star} / \mathrm{rod} / \mathrm{s}$ ) presented for $1 \mathrm{~s}$. A subset of recorded cells were only weakly activated by this stimulus; upon exposure to a $100-\mu \mathrm{m}$-diameter spot, RGCs that were previously attenuated now offered substantially larger responses (see Fig. 2). This suggested that these might be cells with small RFs and strong surround suppression. Therefore we recharacterized the light responses of these RGCs with a $100 \mu \mathrm{m}$ light spot (Fig. 1I-P). Consistent with their poor response to $200 \mu \mathrm{m}$ spots and with other results shown below (see Figs. 7, 8), HD RGCs were only very weakly activated by other stimuli classically used in largescale surveys of RGC responses, including all full-field stimuli (Fig. 2), flickering checkerboards, or drifting gratings (data not shown). Because their small dendritic fields (Fig. $1 A-C$ ) imply that they constitute a significant fraction of RGC types in the mouse retina and are perhaps among the most densely populated, we named these cell types HD RGCs based on the structure of pixel densities in modern displays. Considering both the small RF profiles of HD RGCs and their highly selective response profiles to visual stimuli, it is likely that HD RGCs have been heavily under-represented in previous functional classification studies (Farrow and Masland, 2011; Baden et al., 2016).

The three HD RGC types we encountered with our RFcentered $100 \mu \mathrm{m}$ spot stimulus are functionally distinct (as shown quantitatively below), yet they share key physiological and morphological features with LED RGCs. All are ON-OFF cells stratifying predominantly in the middle of the IPL (Fig. $1 E-H$ ), all lack direction selectivity (Fig. 2I), and all exhibit complete suppression of the center response by concurrent surround stimulation (Fig. $2 B-E$; with the exception being the ON response of HD2 RGCs).

Following functional identification, we filled a subset of cells for morphological analysis (see Materials and Methods). Axons projecting toward the optic nerve confirmed the identity of the target cells as RGCs (data not shown). HD1 and HD2 RGCs had similar dendritic areas and stratification patterns (Figs. 1A,B,E, F, $2 G$; Table 1 ), but differed substantially in their light responses (Fig. $1 I, J, M, N$ ). HD1 RGCs had more sustained responses at light onset than at light offset (Fig. 1I,M). Conversely, HD2 RGCs fired a brief burst of spikes at light onset and a more sustained burst at offset (Fig. $1 \mathrm{~J}, N$ ). In response to a $100 \mu \mathrm{m}$ spot of light from darkness, the UHD RGC fired transient bursts of spikes at light onset and offset nearly equal in number and commonly displayed spike amplitude adaptation (Fig. $1 K, O$ ). The UHD RGC (Fig. $1 C, G, K, O$ ) had the smallest dendritic area of the HD RGCs and among the smallest reported for mouse RGCs (Figs. 1C, 2G; Table 1; Helmstaedter et al., 2013). The majority of its dendrites terminated in the middle of the IPL, but several dendritic tips project into the OFF sublamina (Fig. $1 G$; Helmstaedter et al., 2013, their cell type g36-51).

Our morphological and physiological data from LED RGCs agrees with previously published results from these cells (Zhang et al., 2012). They have a dendritic field smaller than HD1 and HD2 RGCs but larger than UHD RGCs (Fig. 2G; Table 1). They are monostratified in the middle of the IPL like the HD RGCs (Fig. 1H). The LED RGC light response could be distinguished from those of the HD RGCs by its highly latent response, long duration, and lower peak-firing rate (Fig. $1 L, P$ ). Quantitative decision boundaries on parameters of the light responses of these four RGC types set criteria on which they could be unambiguously divided and are described below.

\section{HD RGCs possess small RF centers and strong surround inhibition}

To probe the RFs of the HD RGCs, we presented bright spots from darkness in pseudorandom order, ranging from 10 to $600 \mu \mathrm{m}$ (Fig. $2 A-F$ ). While all HD RGCs indeed had small RFs $(<200 \mu \mathrm{m}$ for the ON or OFF responses, or for both) and strong surround suppression, they differed in several respects. HD1 RGCs had stronger ON responses than OFF responses across all spot sizes (Fig. 2B). HD2 RGCs instead responded more strongly to light offset than to onset (for small spots), and their $\mathrm{ON}$ and OFF responses showed a different pattern of surround suppression. OFF responses of HD2 RGCs were completely suppressed at spot sizes $>400 \mu \mathrm{m}$, but ON responses were never fully suppressed even at spots sizes up to $600 \mu \mathrm{m}$ (Fig. 2C).

UHD RGCs displayed equivalent spike rates at light onset and offset, and were completely suppressed for spots $>275 \mu \mathrm{m}$ (Fig. $2 D$ ). LED RGCs had ON and OFF responses that were also completely suppressed for large spots (Fig. 2E), consistent with pre- 
A

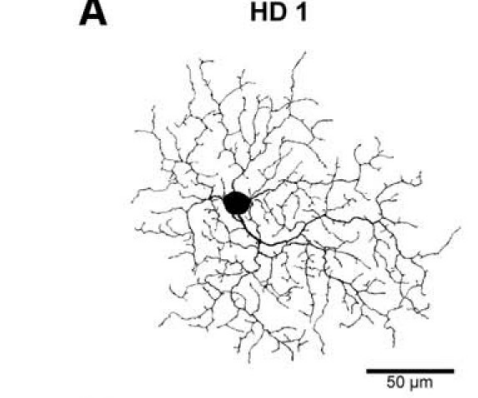

E
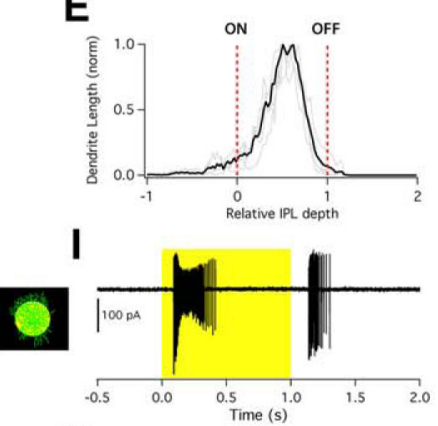

M

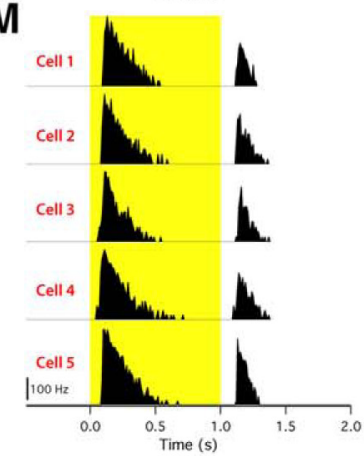

B
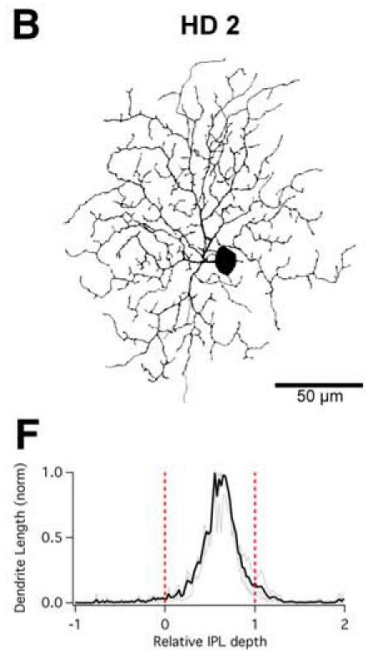

J

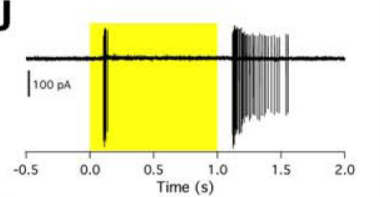

N

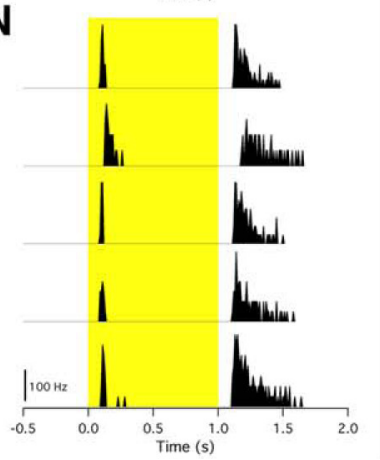

C

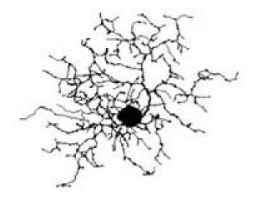

G

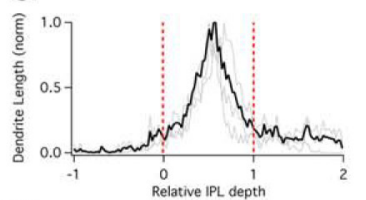

K

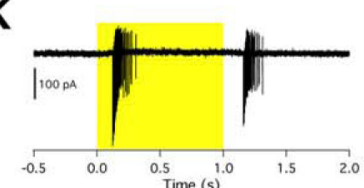

0

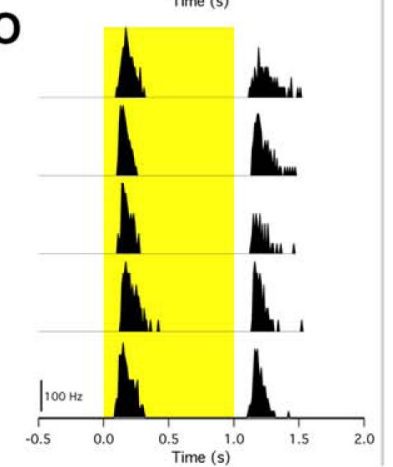

D

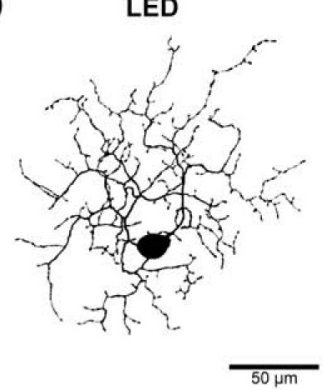

H

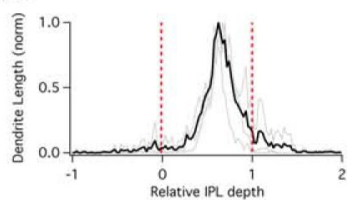

L

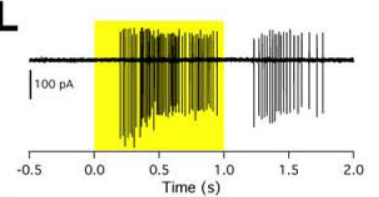

P

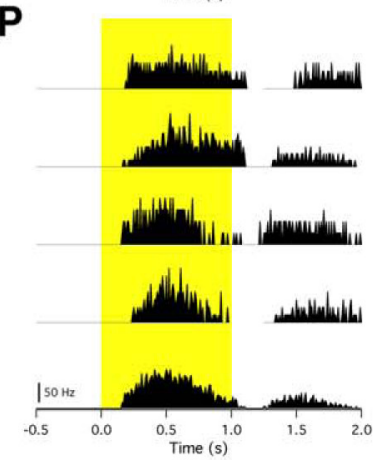

Figure 1. Three novel RGCS and the LED RGC recorded from the mouse retina. Columns represent individual cell types: HD1, HD2, UHD, and LED RGCS. A-D, Fluorescent images (inverted) acquired using a confocal microscope to show the morphology and respective size of representative cells of each type. Scale bars, $50 \mu \mathrm{m}$. $\boldsymbol{E}-\boldsymbol{H}$, Dendritic stratification profiles depicting dendritic length ( $y$-axis) versus IPL depth ( $x$-axis) with dotted red lines marking the locations of $0 \mathrm{~N}(0)$ and $0 \mathrm{FF}$ (1) ChAT bands. Gray lines show raw stratification profiles for a single cell; black line shows multicell average. $I-L$, Single-cell capacitive spike train from cell-attached recording evoked from $100 \mu \mathrm{m} \mathrm{spot} \mathrm{of} \mathrm{light} \mathrm{at} 200 \mathrm{R}^{*} / \mathrm{rod} / \mathrm{s}$ (highlight) from darkness presented to RF center. $\boldsymbol{M}-\boldsymbol{P}$, Peristimulus time histogram of a light step from darkness derived from five different cells of the same type showing the consistency in light responses between cells.

Table 1. Morphological characterization of dendritic area and stratification depth

\begin{tabular}{llllll}
\hline & Area $\left(\mu \mathrm{m}^{2}\right)$ & Diameter $(\mu \mathrm{m})$ & ChAT normalized IPL depth & Eyewire match & Helmstaedter et al. match \\
\hline HD1 & $16,563 \pm 1,131$ & $144 \pm 5(12)$ & $60 \pm 3 \%(4)$ & 5 si or 5s0 & gc37-46 or gc47-57 \\
HD2 & $16,184 \pm 1,319$ & $143 \pm 6(4)$ & $59 \pm 2 \%(3)$ & 5 si or 5s0 & gc37-46 or gc47-57 \\
UHD & $7828 \pm 1,043$ & $99 \pm 7(4)$ & $59 \pm 5 \%(3)$ & 5 ti & gc36-51 \\
LED & $13,642 \pm 1,210$ & $131 \pm 6(4)$ & $66 \pm 2 \%(3)$ & 51 & $\mathrm{gc44-52}$ \\
\hline
\end{tabular}

Area is presented in $\mu \mathrm{m}^{2}$ from images acquired using either confocal or multiphoton fluorescence imaging. Possible matches to morphological identification of EM reconstructions of RGCs in the mouse retina presented on museum.eyewire.org. Numbers in parentheses indicate number of cells.

vious results (Zhang et al., 2012). Suppression in HD RGCs and LEDs was stronger than in another well known ON-OFF RGC type, ON-OFF DS RGCs (Fig. 2F). The spot size necessary to elicit a maximal $O N$ response is one measure of the RF center size. Figure $2 \mathrm{H}$ shows this measurement for each RGC type. UHD RGCs had the smallest RFs ( $89 \pm 3 \mu \mathrm{m}, n=33$ ) among the four non-DS ON-OFF RGCs presented here $\left(p<10^{-5}\right.$ for all pairwise comparisons, Kolmogorov-Smirnov test).

In general, RGC density increases and dendritic arbor size decreases toward retinal locations with higher sampling frequency, such as the fovea in primates and the area centralis in carnivores
(Wässle and Boycott, 1991). A recent study showed that $\alpha$ RGCs displayed a density gradient across the retina, nasal to temporal, that could enhance sampling in frontal visual fields (Bleckert et al., 2014). In our current study, however, the dendritic diameter for HD RGCs showed little variance (Fig. 2G; Table 1). We also observed little dependence of RF size on position in the retina for any of the HD RGCs (Fig. 2L-N). Among a sample of $36 \mathrm{HD} 1,13 \mathrm{HD} 2$, and 18 UHD RGCs, we measured only one significant trend of RF size versus retinal location. The RFs of HD2 RGCs had a small but significant tendency to increase with eccentricity (Fig. $2 M ; r^{2}=0.44, p=$ $0.02, \chi^{2}$ test). All other comparisons of HD RGC RF size versus 
A

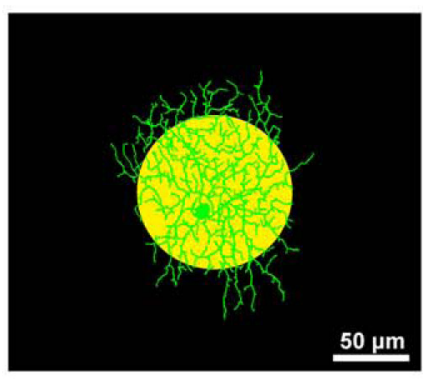

D

UHD

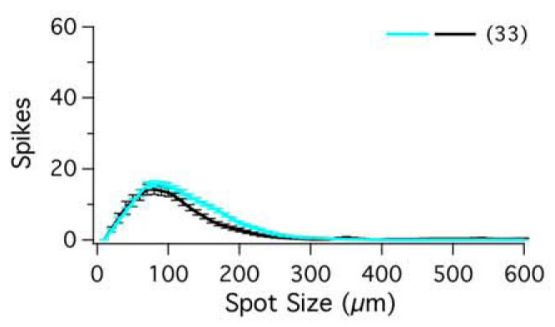

G

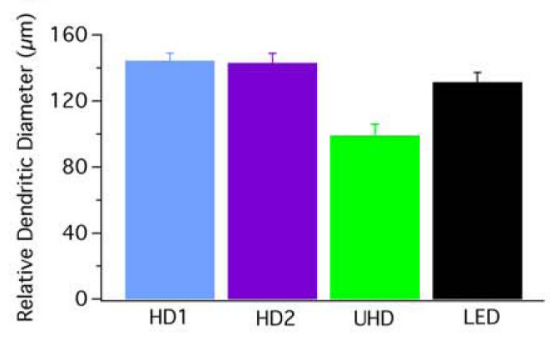

B

HD 1

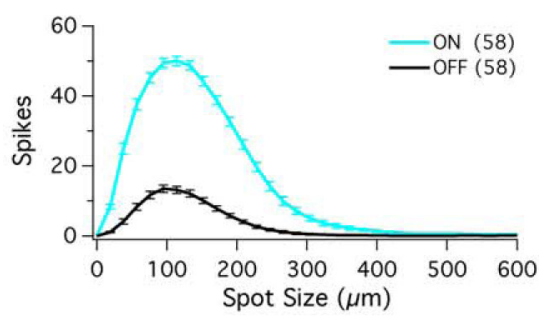

E

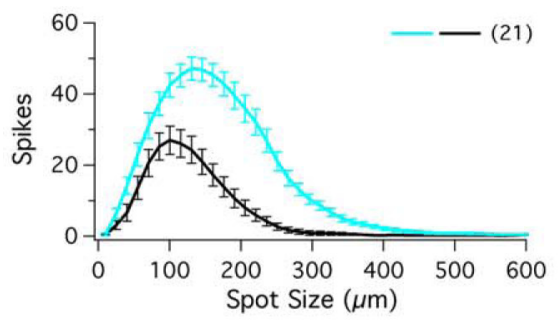

H

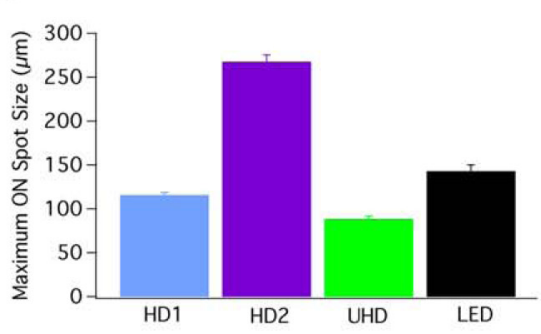

C

HD 2

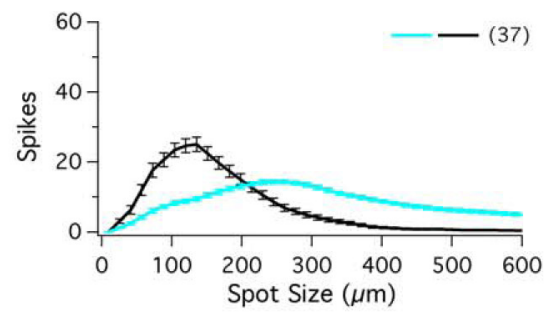

F

ON-OFF DS

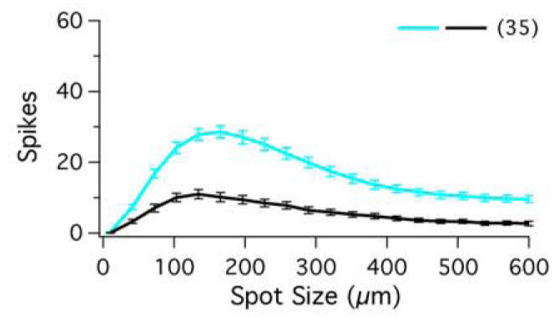

I

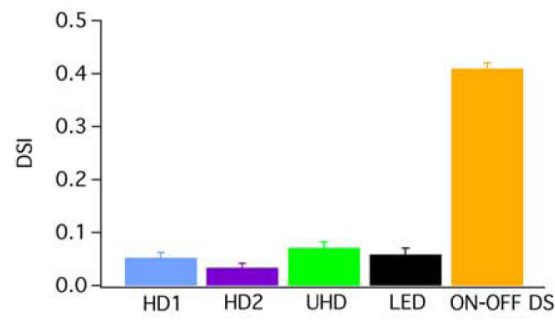

J

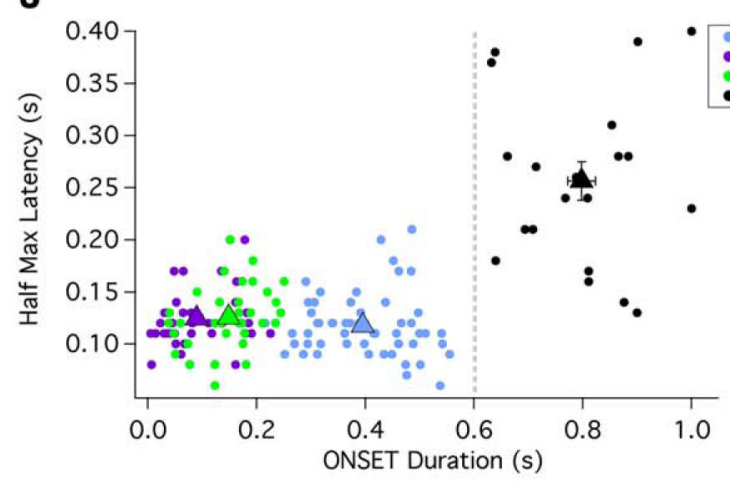

K
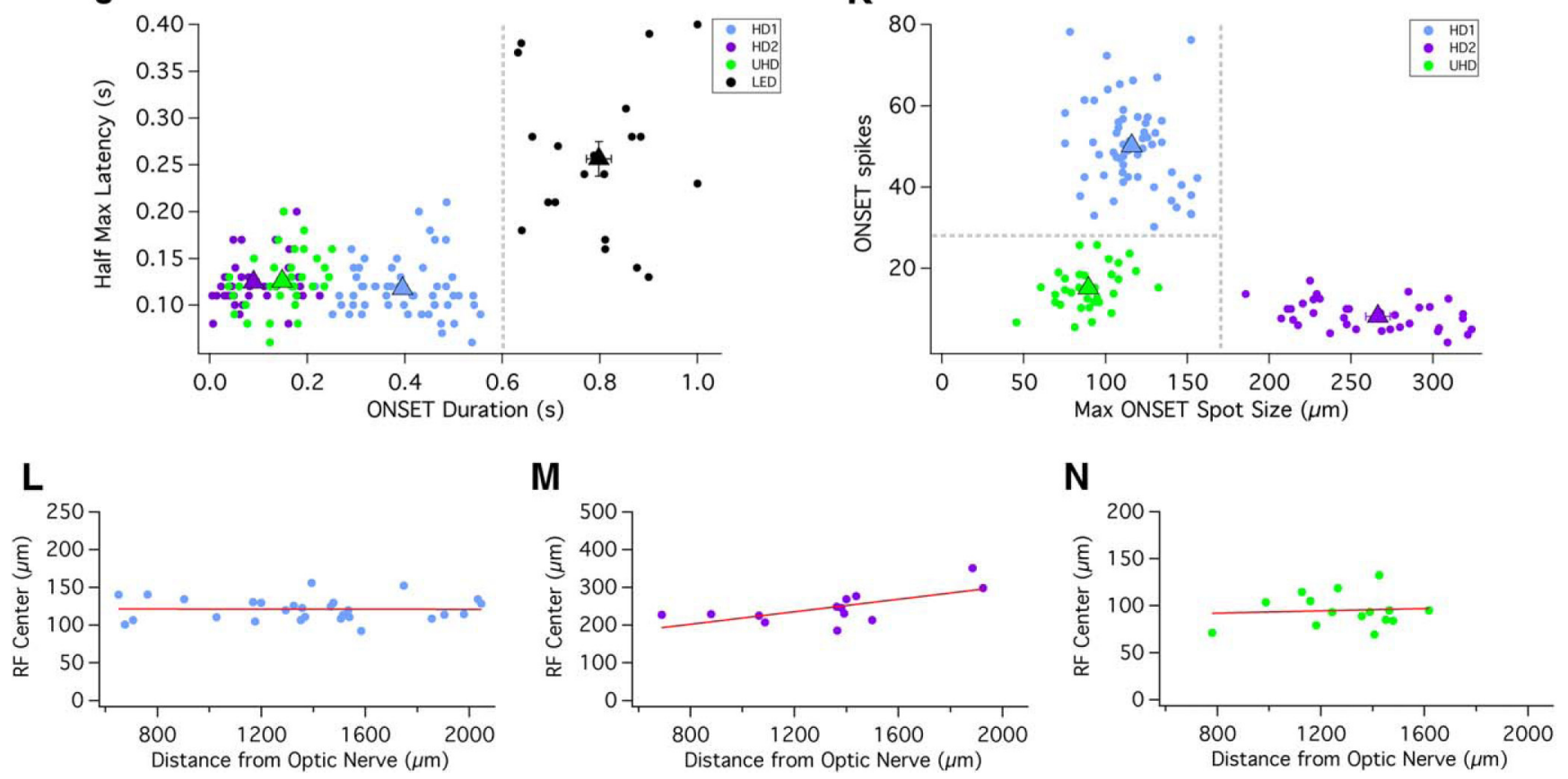

Figure 2. HD cells possess small RF centers and strong surround suppression. $A$, Schematic depicting a $100 \mu \mathrm{m}$ spot of light at $200 \mathrm{R}^{*} / \mathrm{rod} / \mathrm{s}$ (yellow spot) presented from darkness. Scale bar, $50 \mu \mathrm{m} . \boldsymbol{B}-\boldsymbol{F}$, Bright spots of multiple sizes (ranging from 10 to $600 \mu \mathrm{m}$ ) presented to RF center of (B) HD1, (C) HD2, (D) UHD, (E) LED, and (F) ON-OFF DS RGCS. ON (cyan) and OFF (black) responses elicited from bright spots from dark background. G, Bar graph depicting the relative dendritic diameter for HD1 (blue), HD2 (purple), UHD (green), and LED RGCS (black). $\boldsymbol{H}$, Bar graph depicting the relative spot size that generates maximal response for HD1 (blue), HD2 (purple), UHD (green), and LED RGCs (black) during presentation of spots of multiple sizes from darkness. I, Bar graph of the average direction selectivity index (DSI) for HD1 (blue), HD2 (purple), UHD (green), LED (black), and ON-OFF DS RGCs (orange). Error bars are SEM across recorded cells. J, Scatter plot of response duration versus latency (100 $\mu \mathrm{m}$ spots; see Materials and Methods) for the ON responses of the three HD RGCs and the LED. A boundary of $0.6 \mathrm{~s}$ response duration (Figure legend continues.) 
A

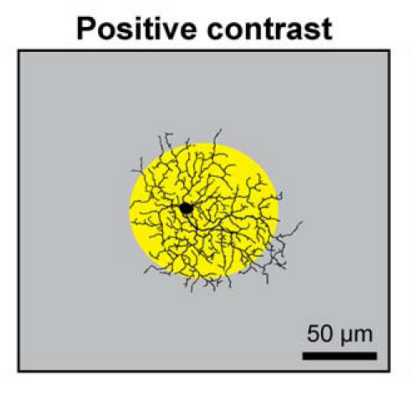

B

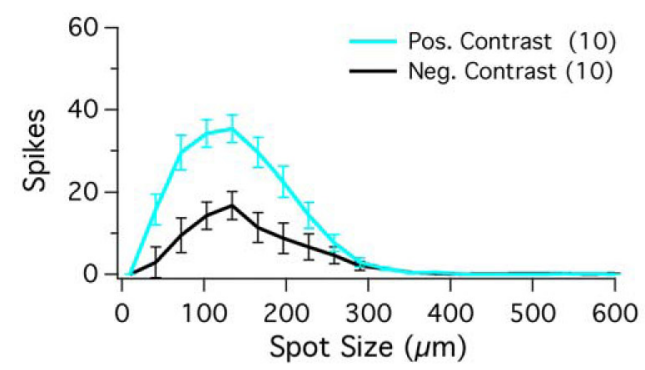

D

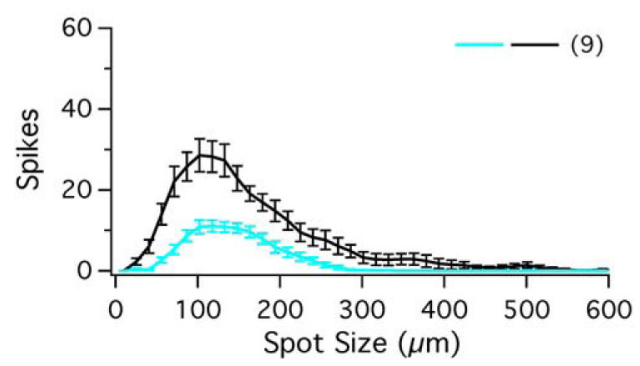

Negative contrast

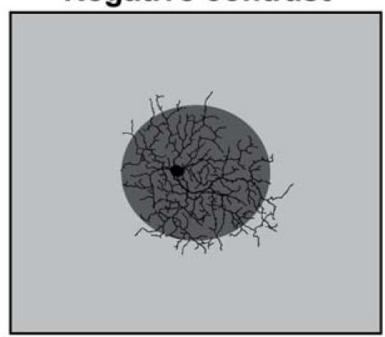

C

HD 2

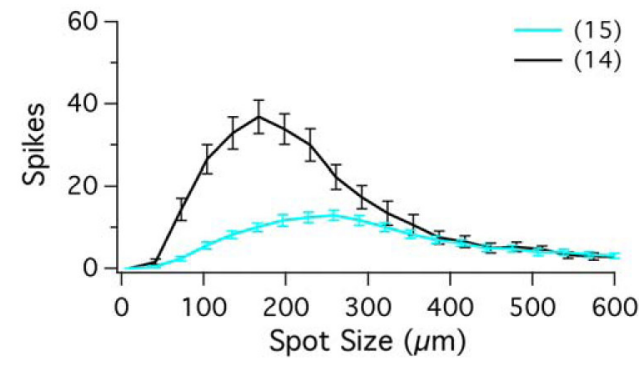

E

\section{LED}

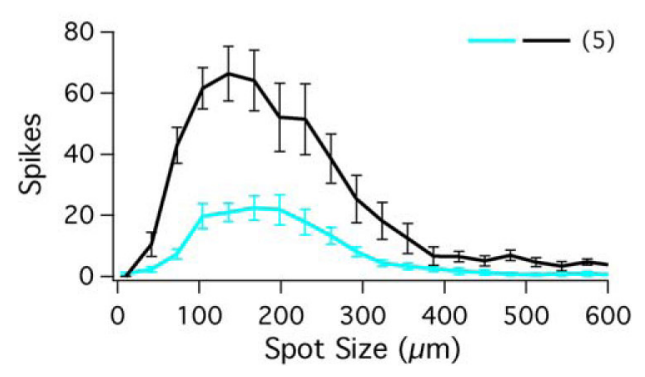

Figure 3. RF structure of HD RGCs in photopic conditions. $A$, Left, Schematic of positive-contrast stimuli for spots of multiple sizes of bright spots from background mean illumination ( $R^{*}$ values provided in text). $\boldsymbol{A}$, Right, Schematic of negative-contrast stimuli for spots of multiple sizes of dark spots ( $0 \mathrm{R}^{*} / \mathrm{rod} / \mathrm{s}$; dark gray spot) from background mean illumination. Scale bar, $100 \mu \boldsymbol{m}$. $\boldsymbol{B}-\boldsymbol{E}$, Positive-contrast and negative-contrast spots of multiple sizes (ranging from 10 to $600 \mu \mathrm{m}$ ) presented to RF center for (B) HD1, (C) HD2, (D) UHD, and (E) LED RGCs. Positive-contrast responses (ON) are shown in cyan and negative-contrast responses (OFF) are shown in black.

eccentricity, dorsoventral position, or nasotemporal position yielded insignificant trends $(p>0.1)$.

Because the HD RGCs are functionally ON-OFF RGCs, we wanted to measure surround suppression in both ON and OFF pathways at equivalent contrasts. We repeated the measurements of the RF profiles under photopic conditions $\left(1000 \mathrm{R}^{\star} / \mathrm{rod} / \mathrm{s}\right)$ using spots of both $100 \%$ positive $\left(2000 \mathrm{R}^{\star} / \mathrm{rod} / \mathrm{s}\right)$ and $100 \%$ negative $\left(0 \mathrm{R}^{\star} / \mathrm{rod} / \mathrm{s}\right)$ Weber contrast (Fig. $\left.3 A\right)$. Two recent studies have identified changes in ON-OFF balance in RGCs with mean illumination (Pearson and Kerschensteiner, 2015; TikidjiHamburyan et al., 2015), and one of these studies argued that key feature selectivity, like direction and orientation selectivity, is retained despite changes in $\mathrm{ON}-\mathrm{OFF}$ balance (Pearson and Ker-

(Figure legend continued.) (dashed gray line) separates LEDs from HD RGCs. $\boldsymbol{K}$, Scatter plot of maximum onset response spot size versus number of spikes at that spot size for the three HD RGCs. Decision boundaries of $175 \mu \mathrm{m}$ and 28 spikes (dashed gray lines) place each HD RGC type in its own quadrant. For both scatter plots, each dot represents the coordinates of a single recorded cell; triangles represent mean of each individual cell type with SEM. Scatter plots for $(\boldsymbol{L})$ HD1, (M) HD2, and ( $\boldsymbol{N}$ ) UHD RGCs display the maximal ON spot size corresponding to the size of the $R F$ center versus retinal eccentricity (distance from optic nerve head). schensteiner, 2015). We compared the results obtained in darkness and from this photopic background to determine whether HD RGCs alter ON-OFF balance with light level and whether they retain their small RFs and strong surround suppression. Both HD1 and HD2 RGCs conserved their response polarity between spots of light presented from darkness and spots of positive and negative contrast presented under mean photopic conditions (Fig. $3 B, C$ ). HD1 remained ON-dominant in both conditions, while HD2 remained OFF-dominant. In photopic conditions, the OFF response of HD2 RGCs was never fully suppressed, as it was in darkness (Figs. 1C, 3C). The transition to photopic light levels, however, altered the ON-OFF balance in both UHD and LED RGCs; both cell types became OFF-dominant (Fig. 3D,E). Despite the small alteration in RF size, all three HD RGC types and LED RGCs retained small RFs and strong surround suppression in the photopic range.

\section{HD RGCs are distinct types}

Of all five ON-OFF RGC types targeted for RF profiling above, only ON-OFF DS cells showed preference for motion direction (Fig. 2I). Following the presentation of a light bar moving in 12 directions from darkness, the direction selectivity index for 


\section{HD 1}

A

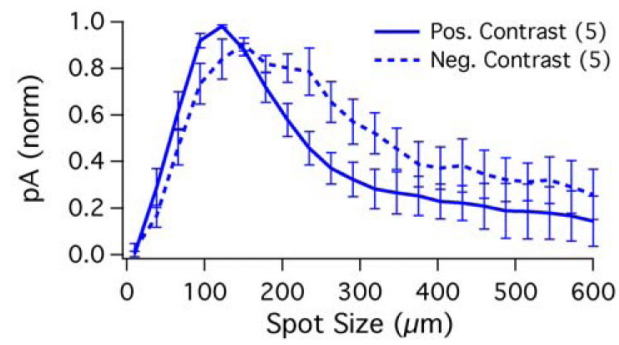

C

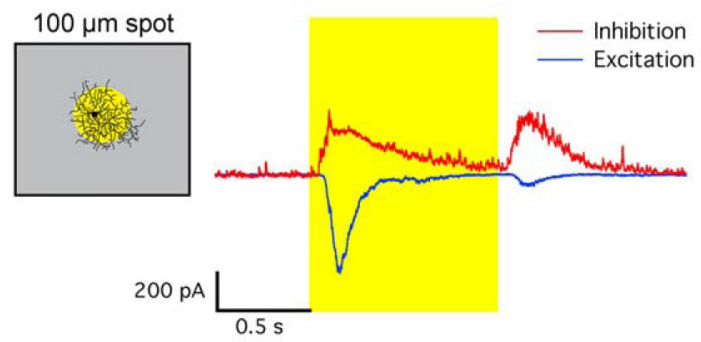

E

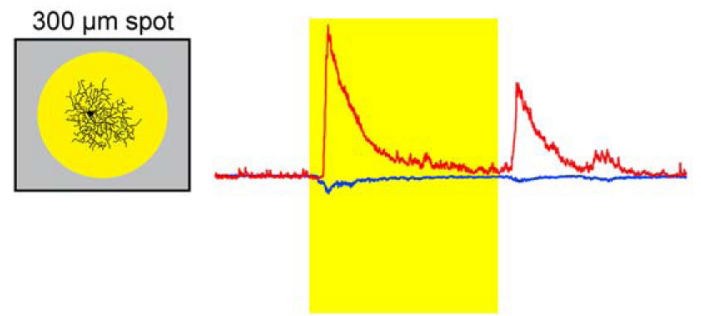

B

Inhibition

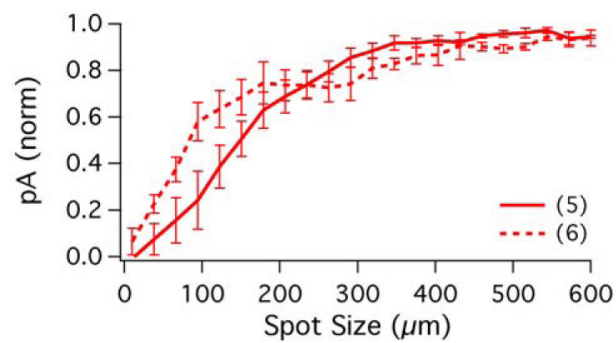

D

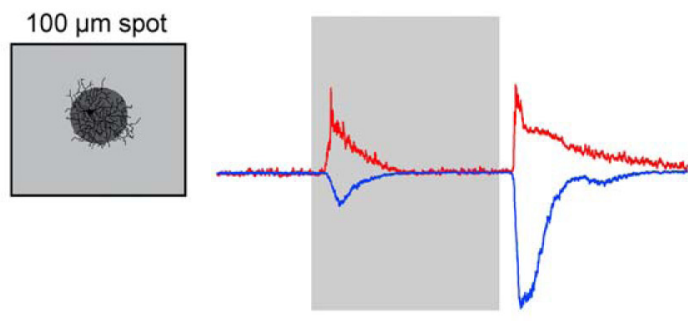

F

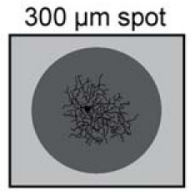

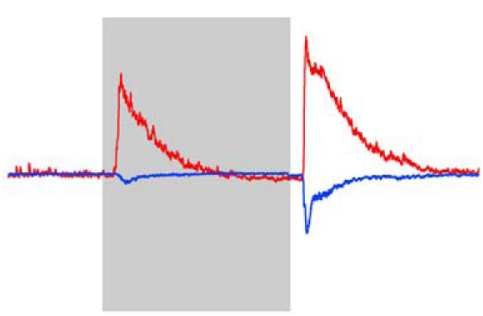

Figure 4. Excitatory and inhibitory currents of HD1 RGCs. $\boldsymbol{A}, \boldsymbol{B}$, Whole-cell patch-clamp recordings from HD1 RGCs measured in voltage clamp to isolate excitatory $(\boldsymbol{A})$ and inhibitory $(\boldsymbol{B})$ currents. $0 N$ response (cyan) and OFF response (black) elicited from bright or dark spots of multiple sizes from background mean light level. $C-F$, Representative current traces for spots of positive or negative contrast of 100 and $300 \mu \mathrm{m}$. Schematics show the spot size and contrast level at which visual stimuli were presented for the corresponding representative traces.

ON-OFF DS cells was highly DS $(0.41 \pm 0.01 ; n=120)$, while the HD1 $(0.05 \pm 0.008 ; n=35)$, HD2 $(0.04 \pm 0.007 ; n=20)$, UHD $(0.07 \pm 0.01 ; n=11)$, and LED RGCs $(0.06 \pm 0.01 ; n=6)$ were not DS.

Despite their similarities, the four non-DS ON-OFF RGCs exhibiting small RF centers could be unambiguously distinguished from each other physiologically. To separate LEDs from HD RGCs, we used the latency and duration of the spike response to a $100 \mu \mathrm{m}$ light spot (Fig. $2 J$ ). The long duration of LED responses was sufficient for classification by dividing cells into groups according to duration: $>0.6 \mathrm{~s}$ (LEDs) and $<0.6 \mathrm{~s}$ (HD RGCs). To classify the three HD RGC types, we used optimal spot size and maximal response amplitude parameters (Fig. $2 K$ ). Separation between these three types was even greater when assessed in higher-dimensional space (data not shown), but the twodimensional space shown in Figure $2 K$ was sufficient to establish strict criteria for functional classification (dashed lines). Independent measures of morphology, RF profiles, synaptic currents, speed sensitivity, and motion sensitivity (Figs. 1-6, 8, 9) confirmed our functional classification. See Discussion for additional comparisons with known RGC types and strategies for functional targeting of HD RGCs for future studies.
Circuit mechanisms of surround suppression in HD RGCs

Several circuit mechanisms contribute to surround suppression in RGCs, including horizontal cell feedback to photoreceptors in the outer retina (Thoreson and Mangel, 2012) and amacrine cell inhibition in the inner retina through both presynaptic inhibition onto bipolar cell terminals and postsynaptic inhibition directly onto RGCs (Grimes, 2012; Masland, 2012a). We hypothesize that the strong surround suppression observed in HD RGCs suggests an origin from amacrine cell inhibition (either presynaptic or postsynaptic) rather than that originating from horizontal cells, which would provide similar surround suppression to all RGCs. To explore the mechanisms of surround suppression in HD RGCs, we measured currents from these RGCs using wholecell voltage clamp following cell-type identification in cellattached configuration. By voltage-clamping these cells at the inhibitory and excitatory reversal potentials, we isolated and quantified the excitatory and inhibitory currents onto the three HD RGC types.

HD1 RGCs received excitatory input from both spots of positive and negative contrast (Fig. 4A). For positive contrast, a spot size of $127 \pm 4 \mu \mathrm{m}$ elicited a maximal excitatory inward current of $-409 \pm 48 \mathrm{pA}(n=5)$, while for negative contrast a spot size of 


\section{HD 2}

A

\section{Excitation}

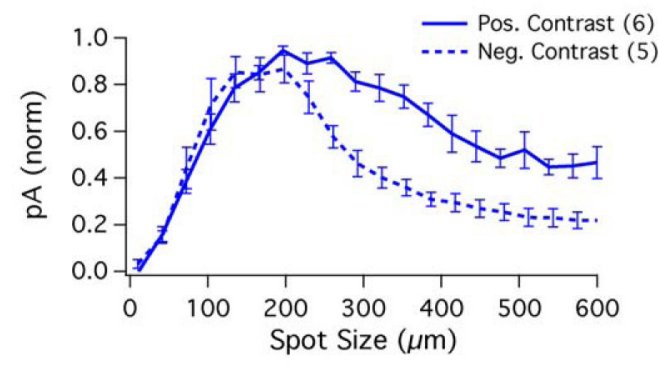

C

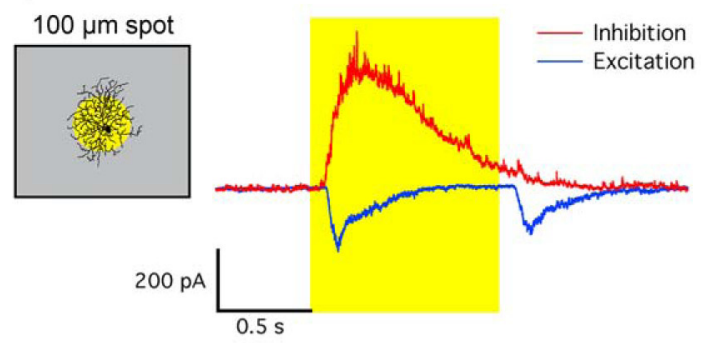

E

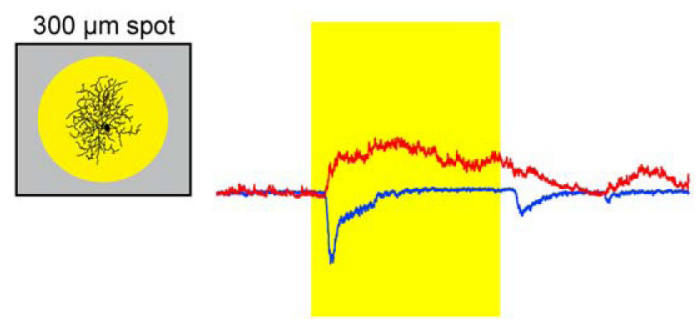

B

Inhibition

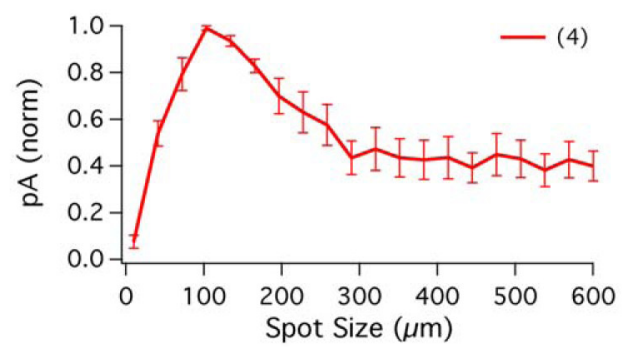

D
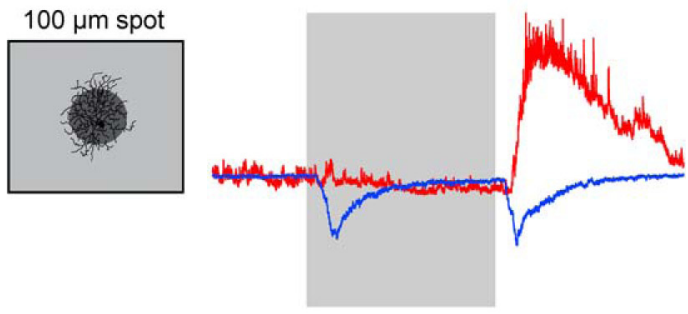

F

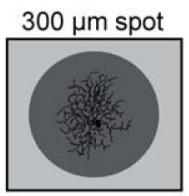

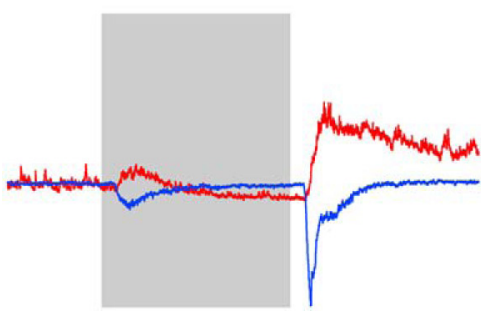

Figure 5. Excitatory and inhibitory currents of HD2 RGCs. $\boldsymbol{A}, \boldsymbol{B}$, Whole-cell patch-clamp recordings from $\mathrm{HD} 2 \mathrm{RGCs}$ measured in voltage clamp to isolate excitatory $(\boldsymbol{A})$ and inhibitory $(\boldsymbol{B})$ currents. No inhibition was measured to negative-contrast stimuli. ON response (cyan) and OFF response (black) elicited from bright or dark spots of multiple sizes from background mean light level. $\boldsymbol{C}-\boldsymbol{F}$, Representative current traces for spots of positive or negative contrast of 100 and $300 \mu \mathrm{m}$. Schematics show the spot size and contrast level at which visual stimuli were presented for the corresponding representative traces.

$153 \pm 14 \mu \mathrm{m}$ produced a maximal excitatory current of $-552 \pm$ $70 \mathrm{pA}(n=5)$. These excitatory currents were highly suppressed during presentation of larger spots, with a higher degree of suppression measured for spots of positive contrast $(86 \pm 8 \%)$ than for spots of negative contrast ( $75 \pm 8 \%$; paired one-sided $t$ test, $p<0.02)$. HD1 RGCs also received strong inhibitory currents to both spots of positive and negative contrast (Fig. 4B). For positive contrast, a spot size of $551 \pm 39 \mu \mathrm{m}$ elicited a maximal inhibitory current of $672 \pm 149 \mathrm{pA}(n=5)$, while for negative contrast a spot size of $499 \pm 47 \mu \mathrm{m}$ produced a maximal inhibitory current of $489 \pm 110 \mathrm{pA}(n=5)$. Unlike the excitatory currents, inhibition showed no surround suppression, instead increasing for spots $\leq \sim 300 \mu \mathrm{m}$ before reaching a plateau. Representative traces of HD1 excitatory and inhibitory currents in response to 100 or $300 \mu \mathrm{m}$ spots of either $100 \%$ positive or $100 \%$ negative contrast stimuli can be seen in Figure $4 C-F$.

At smaller spot sizes, the HD1 RGC received substantial ON and OFF excitation unrivaled by weak inhibition, allowing the cell to spike readily to both positive and negative contrast spots $<300 \mu \mathrm{m}$ in diameter (Fig. $4 A$ ). At spot sizes $>\sim 300 \mu \mathrm{m}$, the interplay between reduced excitatory current and increased inhibitory current led to the full suppression of spike re- sponses. The inhibitory current steadily increased over the range of spot sizes where we observed surround suppression in the spike response. These results show that both presynaptic (onto bipolar cell terminals) and postsynaptic (onto RGC dendrites) forms of inhibition contribute to surround suppression in HD1 RGCs.

HD2 RGCs also received excitatory input from both spots of positive and negative contrast (Fig. $5 A$ ). For positive contrast, a spot size of $215 \pm 21 \mu \mathrm{m}$ elicited a maximal excitatory inward current of $-210 \pm 33 \mathrm{pA}(n=6)$, while for negative contrast a spot size of $231 \pm 26 \mu \mathrm{m}$ produced a maximal excitatory current of $-494 \pm 96 \mathrm{pA}(n=5)$. Both excitatory currents were suppressed for larger spot sizes, to nearly half for positive contrast (52 $\pm 8 \%)$ and to a larger extent for negative contrast $(78 \pm 4 \%$; paired one-sided $t$ test, $p<0.01$ ). Unlike HD1 RGCs, HD2 RGCs received inhibition only for positive contrasts (Fig. 5B). An optimal spot size of $121 \pm 5 \mu$ m elicited a maximal inhibitory current of $277 \pm 52 \mathrm{pA}(n=4)$. Inhibition in HD2 RGCs showed its own surround suppression, falling $60 \pm 6 \%$ for the $600 \mu \mathrm{m}$ spot. Representative traces of HD2 excitatory and inhibitory currents in response to 100 or $300 \mu \mathrm{m}$ spots of either $100 \%$ positive or $100 \%$ negative contrast stimuli can be seen in Figure $5 C-F$. 


\section{UHD}

A

Excitation

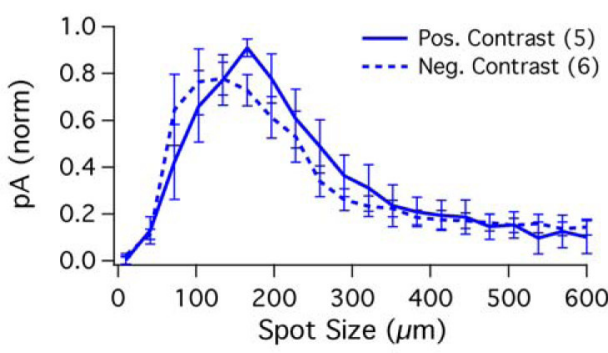

C

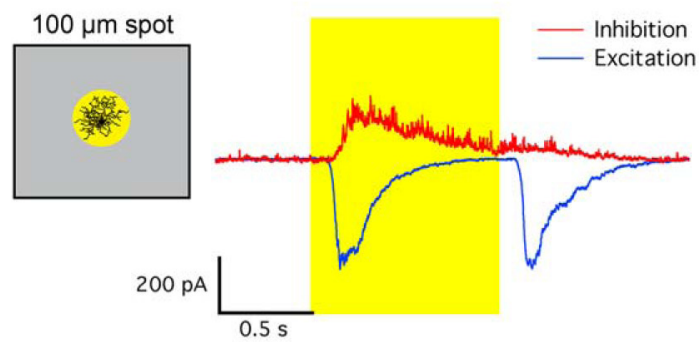

E

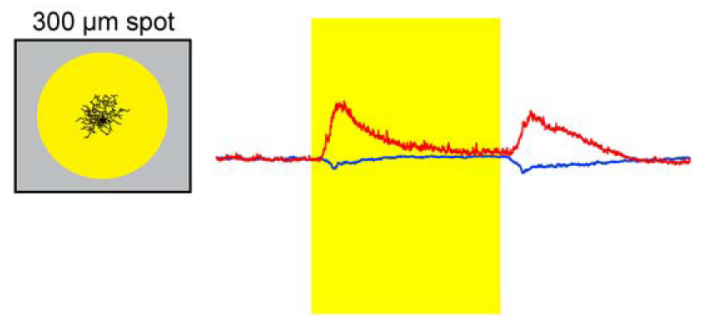

B

Inhibition

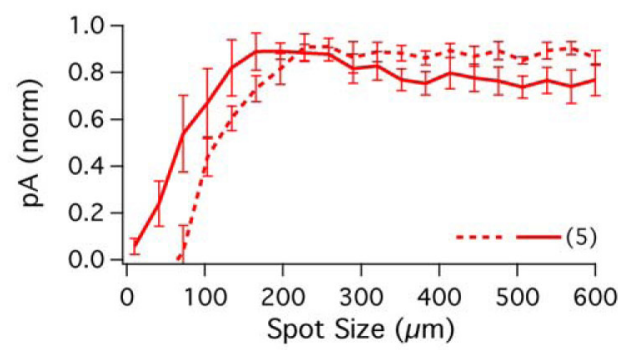

D

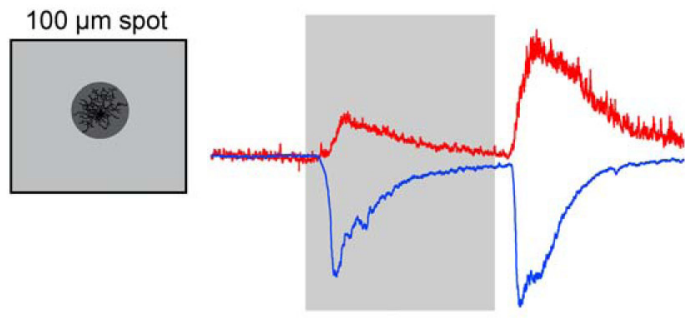

F

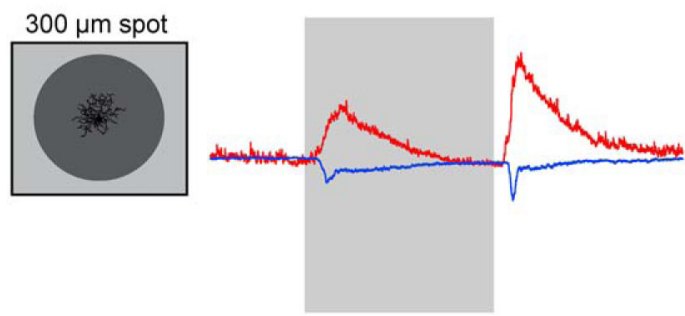

Figure 6. Excitatory and inhibitory currents of UHD RGCS. $\boldsymbol{A}, \boldsymbol{B}$, Whole-cell patch-clamp recordings from UHD RGCs measured in voltage clamp to isolate excitatory $(\boldsymbol{A})$ and inhibitory $(\boldsymbol{B})$ currents. ON response (cyan) and OFF response (black) elicited from bright spots of multiple sizes from background mean light level. $\boldsymbol{C}-\boldsymbol{F}$, Representative current traces for spots of positive or negative contrast of 100 and $300 \mu \mathrm{m}$. Schematics show the spot size and contrast level at which visual stimuli were presented for the corresponding representative traces.

These results show differences in the mechanisms of surround suppression between HD1 and HD2 RGCs. Direct inhibition to the RGC does not appear to play a role in surround suppression in HD2 RGCs because the inhibition itself was suppressed for large positive-contrast spots, and inhibition was completely absent for negative contrasts. Instead, surround suppression in spiking output is controlled by a presynaptic mechanism that reduces excitatory currents for large spots. Surround suppression of excitation was stronger for negative contrasts than for positive contrasts (Fig. 5A), consistent with the spike results (Fig. 3C). While postsynaptic inhibition does not play a role in surround suppression in HD2 RGCs, it may serve to truncate the spike response, contributing to the transient nature of the $\mathrm{ON}$ response (Fig. 1J,N).

UHD RGCs received excitatory input in response to both spots of positive and spots of negative contrast (Fig. 6A). For positive contrast, a spot size of $154 \pm 18 \mu \mathrm{m}$ elicited a maximal excitatory inward current of $-249 \pm 62 \mathrm{pA}(n=5)$, while for negative contrast a spot size of $144 \pm 17 \mu \mathrm{m}$ produced a maximal excitatory current of $-433 \pm 44 \mathrm{pA}(n=5)$. Both excitatory currents were equally suppressed to nearly zero in response to larger spot sizes in positive $(90 \pm 7 \%)$ and negative ( $85 \pm 4 \%$ ) contrast (positive vs negative, $p=0.1$ ). UHD RGCs also re-
Table 2. Overview of the mechanisms involved in the suppression of excitatory and inhibitory currents in HD RGCs and the degree to which these currents are suppressed

\begin{tabular}{clll}
\hline Response & $\begin{array}{l}\text { Excitation } \\
\text { suppression }\end{array}$ & $\begin{array}{l}\text { Inhibition } \\
\text { suppression }\end{array}$ & $\begin{array}{l}\text { Mechanism of surround } \\
\text { suppression }\end{array}$ \\
\hline HD1 & & & \\
ON & $85 \pm 10 \%$ & $5 \pm 1 \%$ & \\
OFF & $75 \pm 8 \%$ & $8 \pm 3 \%$ & Presynaptic and postsynaptic inhibition \\
HD2 & & & \\
ON & $53 \pm 7 \%$ & $60 \pm 6 \%$ & \\
OFF & $78 \pm 4 \%$ & No inhibition & Presynaptic inhibition \\
UHD & & & \\
ON & $90 \pm 7 \%$ & $23 \pm 7 \%$ & \\
OFF & $85 \pm 4 \%$ & $13 \pm 3 \%$ & Strong presynaptic and postsynaptic inhibition \\
\hline
\end{tabular}

ceived strong inhibitory currents for both spots of positive and spots of negative contrast (Fig. 6B). For positive contrast, a spot size of $261 \pm 86 \mu \mathrm{m}$ elicited a maximal inhibitory current of $320 \pm 56 \mathrm{pA}(n=5)$, while for negative contrast a spot size of $289 \pm 81 \mu \mathrm{m}$ produced a maximal inhibitory current of $453 \pm 31 \mathrm{pA}(n=5)$. Inhibitory currents increased with spot size, with $\mathrm{ON}$ inhibition plateauing at $\sim 150 \mu \mathrm{m}$ and $\mathrm{OFF}$ 
A
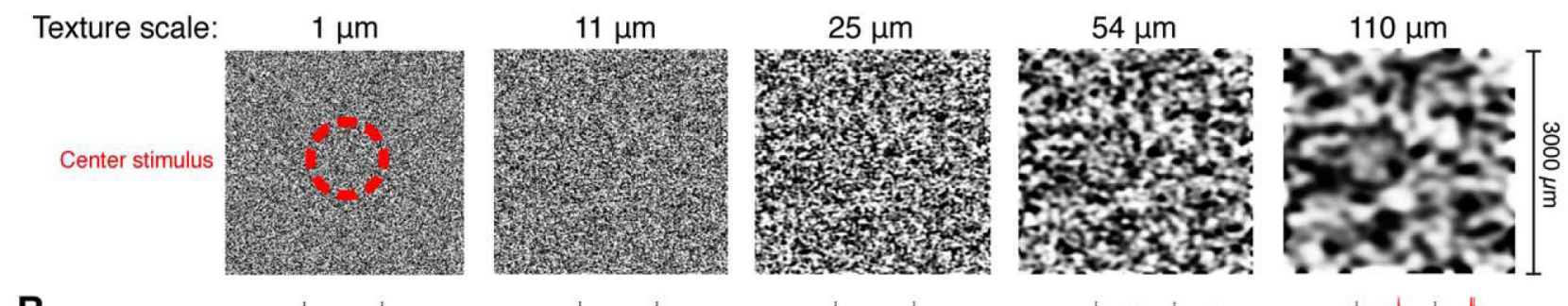

B
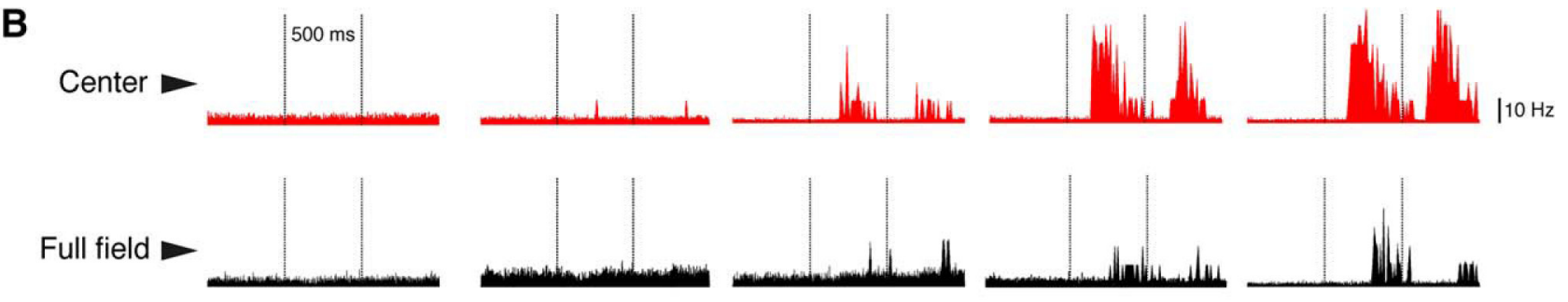

C

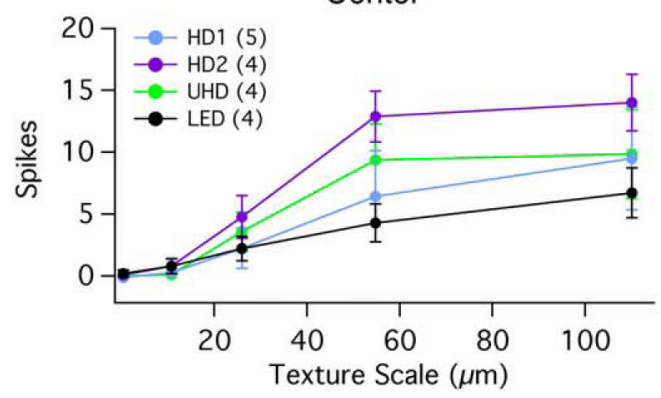

Full field

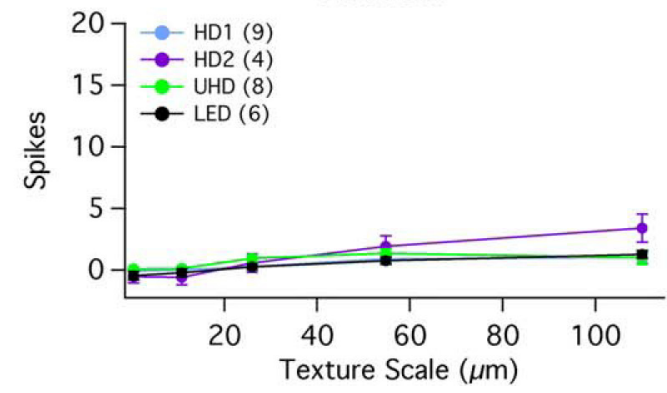

Figure 7. HD RGCs respond to flashed texture stimuli presented to the RF center, but are attenuated to full-field stimuli. A, Graphic representation of the full-field $(3000 \times 3000 \mu \mathrm{m})$ texture stimuli presented to the four cell types tested; each image depicts a different spatial scale $(1,11,25,54$, and $110 \mu \mathrm{m})$. The hash-mark red circle represented the region of stimulus projection when the texture was only presented to the RF center. $\boldsymbol{B}$, Peristimulus time histograms depicting the response to RF center (red traces, top) and full-field (black traces, bottom) textures in a representative HD1 RGC. Flashed textures were presented for a total of $500 \mathrm{~ms}$; stimulus onset and offset are designated by vertical dotted lines. C, Baseline-subtracted spike responses of HD and LED RGCs to textures presented to the RF center (left) or full-field stimuli (right) at different texture scales.

inhibition plateauing at $\sim 250 \mu \mathrm{m}$. Representative traces of UHD excitatory and inhibitory currents in response to 100 or $300 \mu \mathrm{m}$ spots of either $100 \%$ positive or $100 \%$ negative contrast stimuli can be seen in Figure $6 C-F$.

As in HD1 RGCs, both presynaptic and postsynaptic inhibition contributed to spike suppression in UHD RGCs, but both mechanisms were magnified in the UHD RGC. Suppression of excitatory currents was more complete in the UHD RGC for negative contrast ( $85 \pm 4 \%$ vs $75 \pm 8 \%$; $p=0.05$; Fig. $6 A$ ). In response to positive contrast, inhibition increased for smaller spot sizes in UHD RGCs than in HD1 RGCs (Fig. 6B; half maxi$\mathrm{mal}$ spot size $78 \pm 20 \mu \mathrm{m}$ vs $\left.153 \pm 21 \mu \mathrm{m}, p<10^{-4}\right)$. In response to negative contrast, size dependence of inhibition was more similar between these two cell types ( $121 \pm 8 \mu \mathrm{m}$ vs $97 \pm 15 \mu \mathrm{m}, p<$ $10^{-2}$ ). Interestingly, the spatial scale of inhibition for positive versus negative contrast showed a different trend between UHD and HD1 RGCs with positive-contrast inhibition being smaller in spatial scale in UHD RGCs $(p=0.01)$ and negative-contrast inhibition being smaller in spatial scale in HD1 RGCs $(p<$ $10^{-3}$ ). This combination of strong presynaptic and postsynaptic surround suppression contributes to the UHD RGC's extremely small RF (Figs. 2G, 3D). Together these results demonstrate that HD RGCs achieve strong surround suppression in their spike responses through somewhat different combinations of suppression in their synaptic inputs (Table 2).

Thus far we have delivered stimuli to HD RGCs that have uniformly activated either the center alone or the center and surround concurrently. Many RGCs, as has been shown in the ON $\alpha$ RGC (Schwartz et al., 2012), have nonlinear subunits that are the source of excitation to the RF center. Presumably, if the center and surround differed in the spatial scale or linearity of their subunits (with the surround being more linear or having larger subunits than the center), HD RGCs may respond to fullfield "texture" patterns more effectively than full-field uniform light or dark spots. However, upon testing these cells in the same paradigm as Schwartz et al. (2012) performed on the ON $\alpha$ RGC, we found that this was not the case. Surround suppression was equally strong for textures of different scales even though the presentation of textures in the center alone revealed the presence of nonlinear subunits (Fig. 7). A recent report found that subunits in the surround portion of a cell's RF can exhibit nonlinear spatial integration (Takeshita and Gollisch, 2014). In the case of the HD RGCs, the fact that surround suppression is equally strong despite texture scale suggests that RF center and surround have similar nonlinear subunits.

\section{Responses of HD and LED RGCs to objects of differing sizes and speeds}

Since HD and LED RGCs respond preferentially to small objects, and since LED RGCs were additionally shown to respond well to the motion of small objects across their RFs, we tested the selectivity of HD RGCs and LEDs to moving objects. Specifically, we wanted to determine (1) whether the size-selectivity profiles we observed for static objects (Fig. 2) were maintained for moving 
A

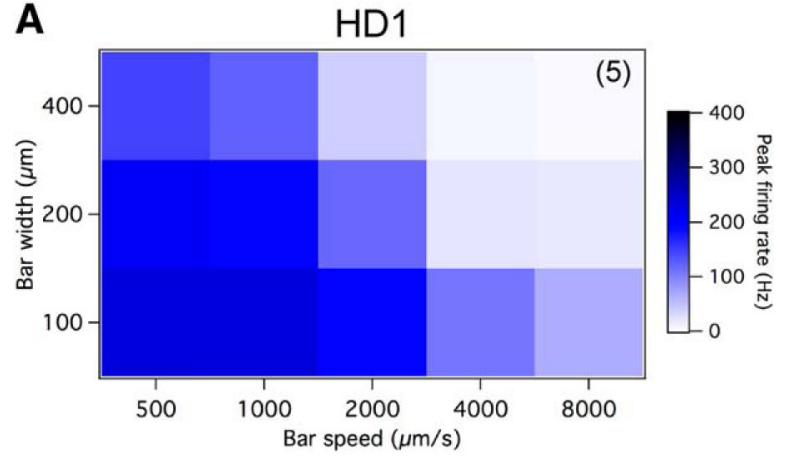

C

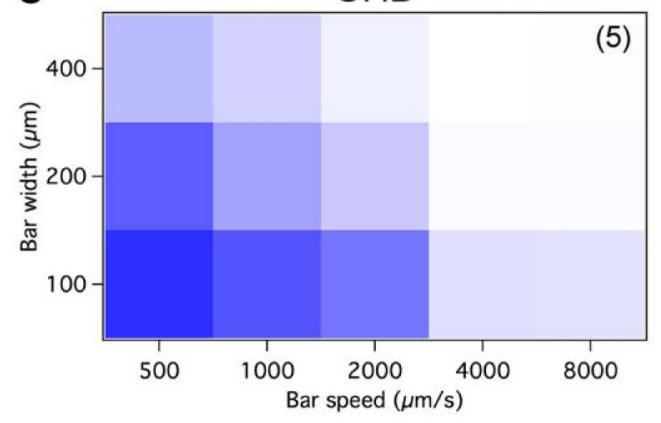

E

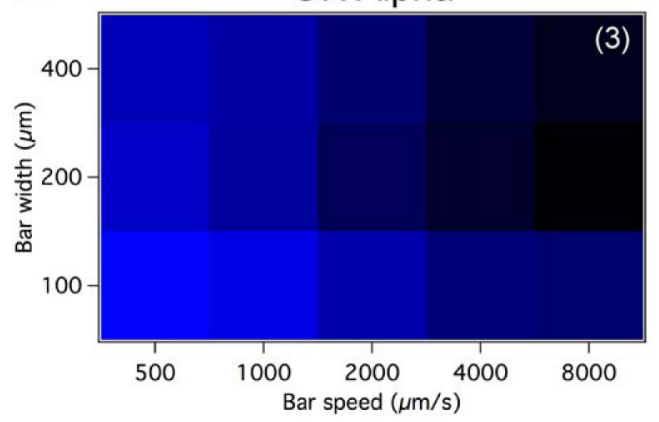

B $\mathrm{HD2}$

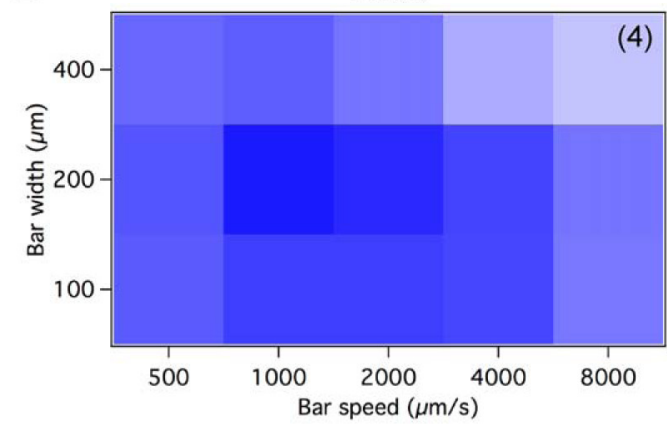

D LED

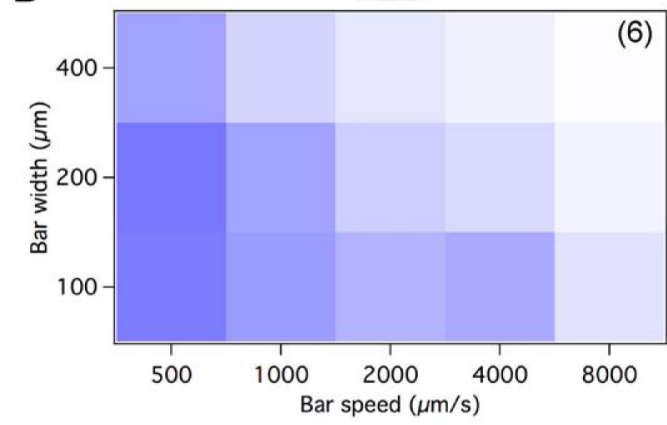

F ON-OFF DS

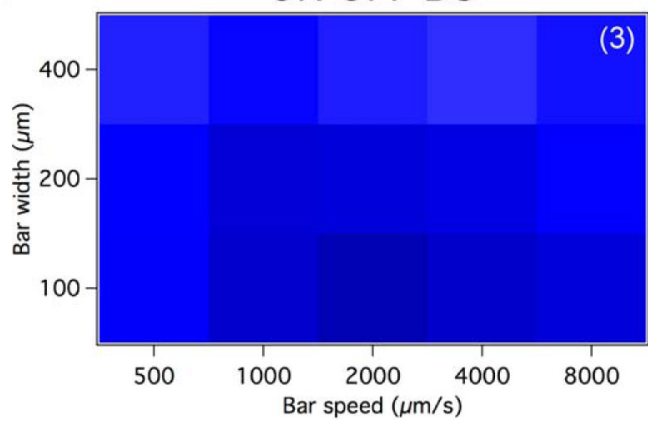

Figure 8. HD RGCs respond to small moving objects with distinct speed and size tuning curves. $\boldsymbol{A}-\boldsymbol{F}$, Moving bars from darkness were modulated in width ( $y$-axis) and speed ( $x$-axis) to determine the characteristic tuning curves for $(\boldsymbol{A}) \mathrm{HD1},(\boldsymbol{B}) \mathrm{HD2},(\boldsymbol{C})$ UHD, $(\boldsymbol{D})$ LED, $(\boldsymbol{E})$ ON $\alpha$, and $(\boldsymbol{F})$ ON-OFF DS RGCs. The color scale displayed adjacent to $\boldsymbol{A}$ is used for each cell type. $N$ values (number of cells) displayed in upper right-hand corner of plots.

objects, and (2) whether speed sensitivity varied among HD and LED RGCs.

The ON response (leading edge of a moving bar from darkness) of the HD1 RGC $(n=5)$ produced the highest firing rate of all cells tested, reaching a rate of $232 \pm 12 \mathrm{~Hz}$ to a $100 \mu \mathrm{m}$ bar moved at $500 \mu \mathrm{m} / \mathrm{s}$, and $230 \pm 18 \mathrm{~Hz}$ to a $100 \mu \mathrm{m}$ bar moved at $1000 \mu \mathrm{m} / \mathrm{s}$ (Fig. 8A). Its response declined as the bar width increased to cover more of the surround portion of the cell's RF and to high-speed bars moving at $\geq 4000 \mu \mathrm{m} / \mathrm{s}$. In stark contrast, the ON response of HD2 $(n=4)$ displayed a nearly uniform activation curve where the peak firing rate was at a bar width of $200 \mu \mathrm{m}$ in diameter and moved at speed of 1000 or $2000 \mu \mathrm{m} / \mathrm{s}$ (Fig. $8 B$ ). This was to be expected, as the ON response of HD2 was never fully suppressed to zero for large stimuli, as was the case for HD1, UHD, and LED RGCs (Figs. 2, 3). Additionally, the HD2 RGC kept up with high-speed moving bars better than the other cell types tested. The ON portion of the UHD RGC $(n=5)$ size and speed tuning curve closely resembled that of HD1, although it never reached HD1 RGCs' peak firing rate of $232 \mathrm{~Hz}$ and displayed a narrower band of activation (activated for thinner bars and slower speeds; Fig. 8C). Much like the HD1, the UHD RGC response declined as bar width increased to cover more of the surround portion of the cell's RF and bar speeds $>2000 \mu \mathrm{m} / \mathrm{s}$. The LED RGC $(n=6)$ again displayed its characteristically low firing rate to bars of 100 or $200 \mu \mathrm{m}$ wide and speeds of $500-$ $1000 \mu \mathrm{m} / \mathrm{s}$; as seen in the HD1 and UHD RGCs, the activation of LED RGCs decreased with increasing bar widths and speeds (Fig. 8D).

To enable our projector to present "smooth motion" stimuli at high speed, we reduced the bit rate to 1 bit from 8 bits. This increased the frame rate from 60 frames/s to 720 frames/s, resulting in an increased number of frames that the bar spent within the cell's RF center in the same amount of time. The modest increases in peak firing rate we observed using this higher frame rate $(\leq 35 \%)$ do not change our interpretation of these results (data not shown). The separately analyzed OFF responses of the three HD RGC types and the LED RGC all showed similar tuning curves; all displayed characteristics much more sensitive to both size and speed, and had lower firing rates than the cell's reported $\mathrm{ON}$ response tuning curve (data not shown). 
A
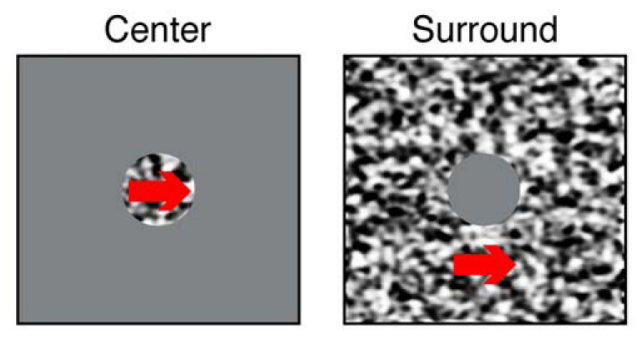
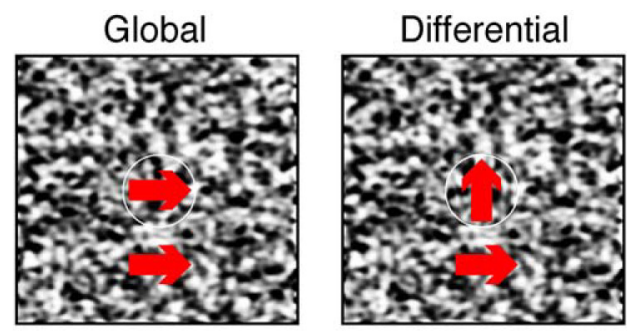

B

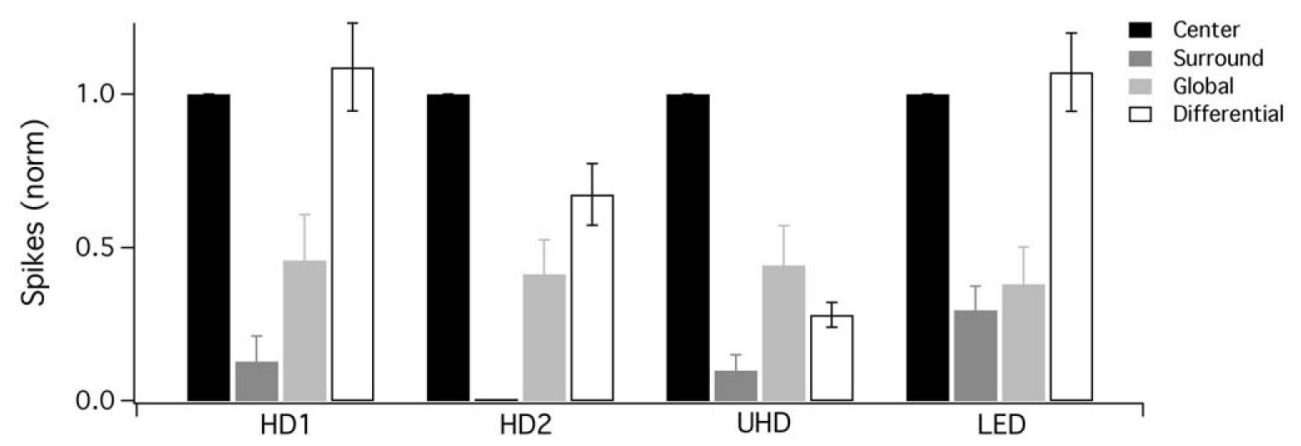

Figure 9. HD RGC responses to object motion. $\boldsymbol{A}$, Schematic depicting the stimuli used to probe responsivity to drifting textures in the (from left to right) RF center, surround only, global, or differential motion between the center and surround portions. Red arrows, placed over the area in which the texture pattern was projected, depict the direction of the texture's drifting motion (200 $\mu \mathrm{m} / \mathrm{s}$ ). Flat gray areas depict that background light level where the texture stimulus was masked. $\boldsymbol{B}$, Bar graph displaying the normalized number of spikes generating by HD1, HD2, UHD, and LED RGCs to drifting textures in the RF center (black), surround only (dark gray), global (light gray), or differential motion in the center and surround portions (white).

We compared the speed and size selectivity of HD and LED RGCs to two well known RGC types: ON $\alpha$ (Fig. $8 E$ ) and ONOFF DS RGCs (Fig. 8F). ON $\alpha$ RGCs showed a tuning profile opposite to HD1, UHD, and LED RGCs; they responded more robustly to large and fast stimuli. Like HD2 RGCs, ON-OFF DS RGCs (probed in their preferred direction) responded nearly uniformly across the range of sizes and speeds we tested.

\section{HD1 and LED RGCs are object-motion-sensitive}

Since object-motion sensitivity is a prominent feature of the LED RGC (Zhang et al., 2012; Kim et al., 2015), we also tested the HD RGCs for this property. We drifted texture stimuli at $1200 \mu \mathrm{m} / \mathrm{s}$ through either the RF center, the surround region, synchronously across both center and surround (global motion), or in a differential fashion where the textures presented to the center and surround were offset $90^{\circ}$ in their direction of motion (Fig. $9 A$; see Materials and Methods). The four RGC types formed two groups based on their responses to differential motion; HD1 and LED RGCs were equally activated to moving textures in the center and to differential motion, and both were substantially suppressed by global motion. Thus, HD1 RGCs can additionally be classified as object-motion-sensitive (Fig. 9B), a property previously described only for LED RGCs in mice (Zhang et al., 2012). HD2 and UHD RGCs were maximally activated by texture movement relegated to the RF center, and were less active in response to surround, global, and differential motion stimuli (Fig. 9B). UHD RGCs had the smallest degree of activation to differential motion of all cell types tested, and all were suppressed by motion in the surround region, by global motion, or by both when compared with motion in the center. Thus, HD2 and UHD RGCs are not object-motion-sensitive, suggesting that the circuits responsible for surround suppression in these RGC types are less sensitive to the precise timing of center and surround features, a mechanism known to be involved in object-motion sensitivity (Baccus et al., 2008; Zhang et al., 2012), than those in HD1 and LED RGCs.
Tracking a moving object with different RGC populations HD and LED RGCs differ in their dendritic diameters (Fig. 1), in their RF sizes and surround suppression (Figs. 2, 3), and in their selectivity profile to the size and speed of moving objects (Fig. 8). We constructed a model mosaic of each of these RGC types, as well as ON $\alpha$ RGCs and ON-OFF DS RGCs, to study how these feature-selectivity differences interact. We measured the performance of a model population of each RGC type in tracking the position of a small moving object. By varying parameters of the model (mosaic spacing, RF size, surround strength, speed selectivity) and the size of the stimulus, we sought (1) to determine which RGC types are best suited to track moving objects of different sizes and (2) to reveal more general principles of how the parameters of a population of RGCs could affect their tracking performance. While additional aspects of the cell responses (e.g., gain control, noise correlations) or of the stimulus (light level, contrast, background) are sure to create additional distinctions between cell types (Fig. 9; see Discussion), we sought to use as few assumptions as possible to explore how the parameters of RGC light responses that we did characterize influence the ability of RGC populations to report object location.

The stimulus in our model was a bright square $(100-400 \mu \mathrm{m})$ on a dark background moving in a quasirandom walk (Fig. 10A; see Materials and Methods). We intentionally ignored the issue of response lag, as motion-anticipation mechanisms have been well studied and depend on gain control at multiple levels in the retinal circuit (Berry et al., 1999; Chen et al., 2013, 2014; Trenholm et al., 2013; Johnston and Lagnado, 2015), and likely elsewhere in the brain (Berry et al., 1999), a subject beyond the scope of the present study.

We computed the center of mass of the firing response of a simulated population of each cell type, and we measured the tracking error as the average displacement between the actual object location and this neural center-of-mass estimate (Berry et al., 1999; Schwartz et al., 2007; Leonardo and Meister, 2013). We 
A

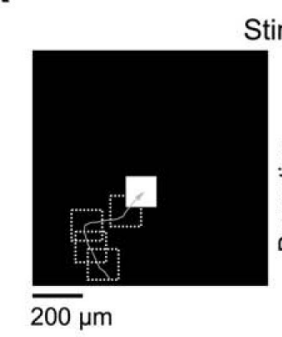

Stimulus
B

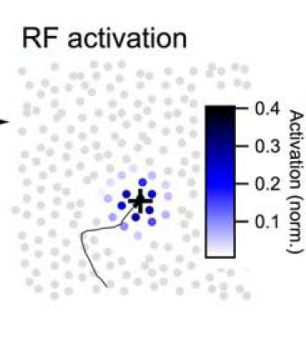

C

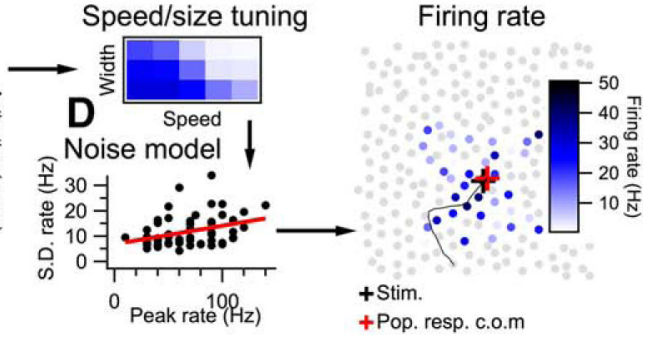

F $100 \mu \mathrm{m}$ square

HD1

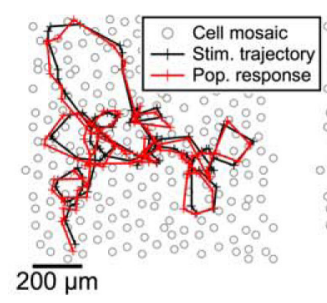

G $400 \mu \mathrm{m}$ square

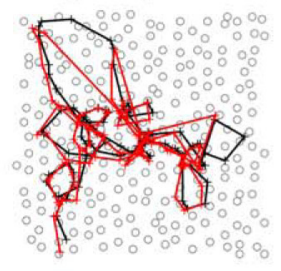

H

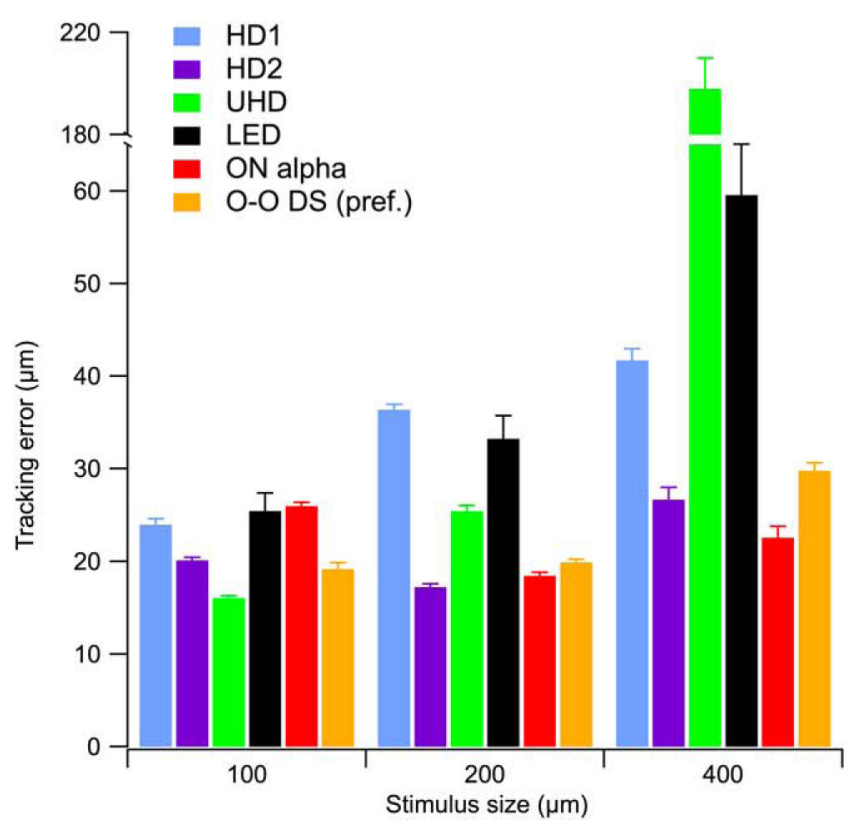

HD2

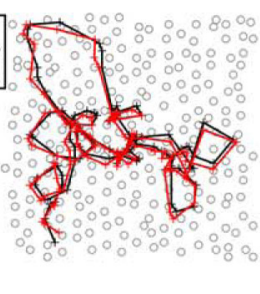

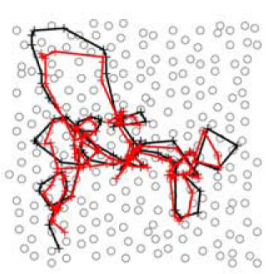

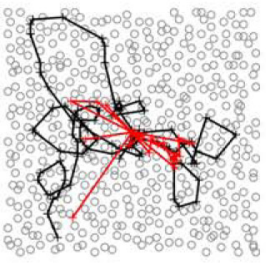

UHD

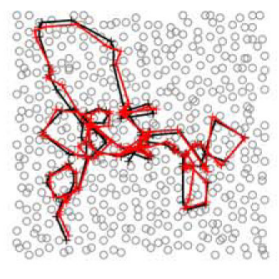

LED

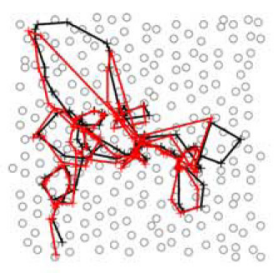

ON alpha

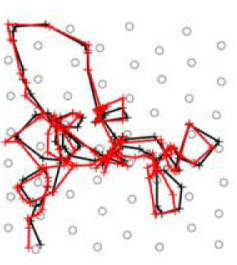

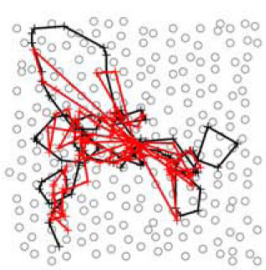

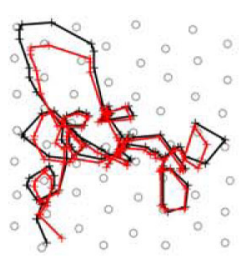

I

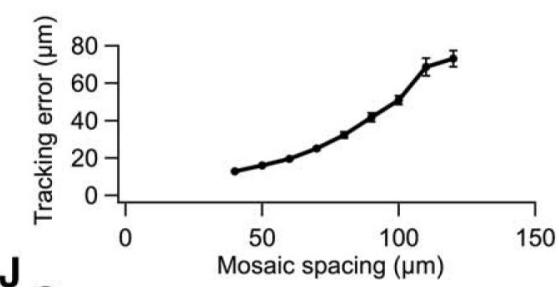

$\mathbf{J}$

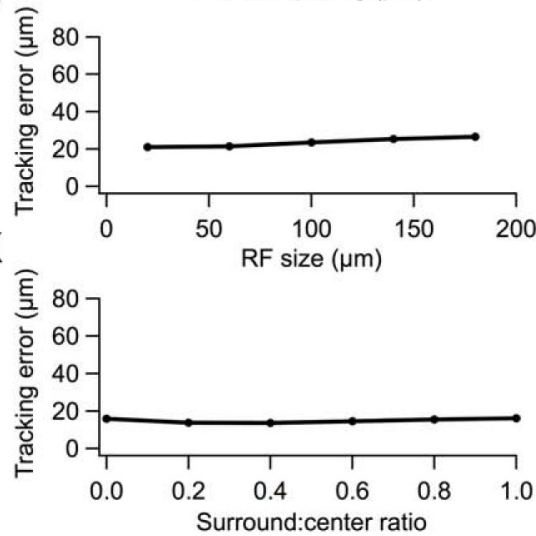

Figure 10. Model of population responses of HD and LED RGCs tracking moving objects. $A$, Left, Schematic of the stimulus showing a white square on a single frame along with its previous trajectory (dotted lines). Right, Velocity distribution for the random walk of the square. $\boldsymbol{B}$, A simulated mosaic of RGCs colored by their degree of RF overlap with the stimulus frame in $\boldsymbol{A}$. C, Speed and size tuning (as in Fig. 8) was applied to the activation pattern in $\boldsymbol{B}$ based on the recent speed of the object. $\boldsymbol{D}$, Noise model for each RGC type. Peak firing rate is plotted against its SD and fit with a line. This model is used to apply noise to each model RGC. $\boldsymbol{E}$, Firing rates for the mosaic in $\boldsymbol{B}$ after incorporating measured speed and size tuning and the noise model. $\boldsymbol{F}$, A model stimulus trajectory (black) and the center of mass of the population response (red) for simulated mosaics of HD1, HD2, UHD, LED, and ON $\alpha$ RGCs for a 100 $\mu \mathrm{m}$ square. Crosses represent $10 \mathrm{~ms}$ time steps. $\boldsymbol{G}$, Sample trajectories as in $\boldsymbol{F}$ for a $400 \mu \mathrm{m}$ square. $\boldsymbol{H}$, Mean tracking error for each RGC type for three different square sizes. ON-0FF DS RGCS (0-0 DS) were measured only in their preferred direction (see text). $I$, Tracking error as a function of mosaic spacing for a simulation in which all other parameters matched the profile of a UHD RGC. $\boldsymbol{J}$, Same as $\boldsymbol{I}$, but varying RF size instead of mosaic spacing. $\boldsymbol{K}$, Tracking error for a UHD population as a function of surround-to-center ratio. Error bars in $\boldsymbol{H}-\boldsymbol{K}$ are $S E M$ across 10 trajectories for each data point. In some cases, the error bars are smaller than the symbols. 
constructed a model mosaic for each cell type based on our anatomical measurements (Table 1) and the well conserved mosaic spacing of RGCs (Wässle et al., 1981; Devries and Baylor, 1997; Masland, 2012b). The model response for each cell in the mosaic was based on our measurements of RF size (Fig. 3), the size and speed tuning curves we measured (Fig. 8), and a noise model extracted from response variability to moving bars (Fig. 10D; see Materials and Methods).

A sample stimulus trajectory and the neural estimate trajectory are shown for model populations of each RGC type for 100 (Fig. 10F) and $400 \mu \mathrm{m}$ (Fig. 10G) squares, and the mean tracking error over 10 simulated trajectories is compared across cell types (Fig. 10H). UHD RGCs showed the smallest tracking error for $100 \mu \mathrm{m}$ objects $(16 \pm 0.2 \mu \mathrm{m})$, but HD2 RGCs were most effective at tracking $200 \mu \mathrm{m}$ objects (error, $17 \pm 0.3 \mu \mathrm{m})$. The general pattern of these results was consistent with the tuning curves measured in Figure 8, but also depended on the mosaic spacing of the cell types inferred from their anatomy (Table 1; Fig. 10I).

UHD and HD2 RGCs outperformed ON $\alpha$ and ON-OFF DS RGCs for small objects, but ON $\alpha$ RGCs were best at tracking 400 $\mu \mathrm{m}$ objects. While the performance differences between the best HD RGCs and the control RGC types were somewhat modest, several caveats suggest that tracking error in a real population of ON $\alpha$ or ON-OFF DS RGCs would be higher than our estimates. First, as pure ON cells, ON $\alpha$ RGCs can only track positive contrast edges, while HD RGCs can track both positive and negative contrast edges. Second, unlike HD RGCs (Fig. 9), ON $\alpha$ RGCs respond almost equally well to local verses global motion because of their weak surrounds (Schwartz et al., 2012). This would create ambiguity for the brain in distinguishing the motion of an object from that of the background. Finally, it is worth noting that our estimates of tracking error for ON-OFF DS cells were based only on their firing rates for stimuli in the preferred direction. As a randomly moving object enters the RF of the RGC in different directions, a single population of ON-OFF DS RGCs would conflate position and direction, and would thus be poorly suited for an object-tracking task.

Varying a single parameter of the model at a time, instead of the multiple parameters required to match the profiles of each RGC type, revealed that tracking error depends more on mosaic spacing than on RF size or the strength of surround suppression (Fig. 10I-K). While surround suppression is, of course, critical to the selectivity of HD RGCs for object motion over global motion, it has little impact on object tracking in our model because the model uses the center of mass of firing of the entire RGC population. More interpretations of the modeling results are described in Discussion.

\section{Discussion}

\section{The first functional characterization of the HD RGCs}

We have described HD RGCs, three new ON-OFF RGC types in the mouse retina. They possess small RFs, strong surround suppression, and selectivity for different sizes, speeds, and patterns of motion (see Table 3). Two small-RF RGCs were recently discovered and named the F-mini ON and F-mini OFF (Rousso et al., 2016). While F-mini cells share the property of small RFs and small dendritic fields, they differ from HD RGCs in dendritic stratification, response polarity (ON or OFF vs ON-OFF), dendritic asymmetry, and direction selectivity. Thus, the HD RGCs, to our knowledge, have not been described functionally despite compelling morphological candidates in the EM data (Helmstaedter et al., 2013).
It is worth noting that despite the expected small variations in experimental conditions, the results obtained from HD and LED RGCs were stable across preparations and across time within a single preparation. To find these cells in future studies without the aid of an HD RGC-specific transgenic marker, the probing visual stimulus must be optimally sized and precisely placed over the RF center. The stimulus must (1) be centered within $\sim 20 \mu \mathrm{m}$ of the RF center to maximally excite the targeted RGC and minimally excite the strong surround region and (2) be close in size to match the RF center (Fig. 2; see Table 3) for each cell type.

\section{HD RGCs have specialized synaptic connectivity}

The functional diversity among the HD RGCs and the LED RGC is notable given their similar morphological properties and stratification profiles (Fig. $1 A-H$; Table 1). UHD RGCs have among the smallest dendritic fields reported for mouse RGCs and, accordingly, show the smallest excitatory RFs (Fig. 6A). While not different in dendritic area (Table 1), HD1 and HD2 RGCs do exhibit different excitatory RF profiles (Figs. 4A, 5A). HD1 RGCs have a smaller excitatory RF and more surround suppression than do HD2 RGCs. This distinction highlights the fact that dendritic morphology alone cannot predict the excitatory RFs of RGCs.

While the coarse division of the IPL into ON versus OFF sublamina (Kolb, 1979) and transient versus sustained subregions within each of the sublamina (Awatramani and Slaughter, 2000; Borghuis et al., 2013) provides a general framework for the synaptic organization of the inner retina, recent evidence points to a finer level of specialization in synaptic contacts even within the same strata of the IPL (Kim et al., 2014; Krishnaswamy et al., 2015; Greene et al., 2016). Differences in the synaptic inputs to the HD RGCs support the notion of fine-scale synaptic specialization in the IPL (Figs. 4-6). Despite their highly overlapping dendritic stratification patterns (Fig. $1 E-H$ ), the three HD RGCs and LED RGCs (Zhang et al., 2012) have distinct patterns of excitatory and inhibitory input with different kinetics, ON-OFF ratios, and size dependence (Figs. 4-6).

With the plethora of amacrine cell types in the retina (Masland, 2001), it is not difficult to imagine that each of these RGC types receives a different complement of inhibitory inputs (see next section). The diversity of bipolar cells, however, is more restricted (Helmstaedter et al., 2013; Greene et al., 2016). The differences in excitatory currents we measured in the HD RGCs could result from a combination of (1) different complements of bipolar cell inputs or even glutamaturgic amacrine cells (Lee et al., 2014; Krishnaswamy et al., 2015), (2) different types of glutamate receptors on the RGCs, and (3) specialized microcircuits at individual bipolar cell terminals (Asari and Meister, 2012). Determining the relative contributions of these three sources of diversity in excitatory drive remains an important open question, and our characterization of the three HD RGCs may facilitate future efforts in this area.

\section{Strong surround suppression uses presynaptic and postsynaptic mechanisms}

The HD RGCs and the LED RGC are specialized (though not necessarily unique) among RGC types in that their spiking can be suppressed completely by concurrent center and surround stimulation. Our results provide insights into the mechanisms responsible for strong surround suppression (Table 2). We show in particular that HD1 and UHD RGCs, which have the strongest surround suppression, employ both presynaptic and postsynaptic mechanisms (Figs. 4, 6), while HD2 RGCs, which have some- 
Table 3. Functional differences between HD and LED RGCs

\begin{tabular}{|c|c|c|c|c|c|c|}
\hline & RF center size & Polarity in darkness & Polarity in photopic range & Response kinetics & Speed sensitivity & Object motion sensitive \\
\hline HD1 & $116 \mu \mathrm{m}$ & ON-dominant & ON-dominant & ON-OFF-transient & Low-medium & Yes \\
\hline HD2 & $268 \mu \mathrm{m}$ & OFF-dominant & OFF-dominant & ON-transient; OFF-sustained & Invariant & No \\
\hline UHD & $89 \mu \mathrm{m}$ & Equal & OFF-dominant & ON-OFF-transient & Very low & No \\
\hline LED & $143 \mu \mathrm{m}$ & ON-dominant & OFF-dominant & ON-OFF-sustained & Low-medium & Yes \\
\hline
\end{tabular}

what weaker suppression, use only a presynaptic mechanism (Fig. 5). Previous work has shown that LED RGCs also use both presynaptic and postsynaptic surround mechanisms, though in that case they were shown to be specialized for the suppression of global motion signals (Zhang et al., 2012; Kim et al., 2015). Employing similar techniques to measure surround suppression in the excitatory and inhibitory currents of other RGC types will provide important comparisons to the surround suppression mechanisms in HD RGCs. Such studies may reveal whether RGCs with weaker surround suppression tend to use either presynaptic or postsynaptic suppression but not both, or whether the degree of suppression in both excitatory and inhibitory currents is lower in those RGCs

\section{Distinct tuning for motion}

The $>30$ RGC types of the vertebrate retina form a distributed representation of ecologically important aspects of visual scenes. Early in the history of studies of RGC responses, the movement of a small spot was assigned a role in prey detection (Lettvin et al., 1959; Zhang et al., 2012). Moving objects varying in speed, size, and contrast elicit a variety of stereotyped behavioral responses in several species (Ishikane et al., 2005; Yilmaz and Meister, 2013; Semmelhack et al., 2014; Shang et al., 2015; Temizer et al., 2015). Here, we augment our understanding of the representation of moving objects in the mouse retina by introducing three new RGC types with properties that suggest a specialization for such stimuli. Strong surround suppression in the HD RGCs endows them with selectivity for objects near their RF size moving on a static (or, for HD1, differential) background. Thus, these cells would be predicted to respond to movements of small objects in the visual scene, but would not be predicted to fire during head or body movements inducing global motion on the retina. Complete suppression of the spike responses for large stimuli makes the HD RGCs different from other well studied RGC types (e.g., $\alpha, \mathrm{ON}-\mathrm{OFF}$ DS, ON DS, orientation selective) in that their firing could specifically encode object motion without the ambiguity of responses to motion of the entire visual field caused by eye, head, or body movements. LED RGCs share similar surroundsuppression properties. So why did the mouse evolve four separate RGC types with similar responses?

Perhaps the answer lies in the distinct tuning of each cell type to particular parameters of motion. Due primarily to their high density inferred from their small dendritic fields, UHD RGCs performed best in the object-tracking model for small $(100 \mu \mathrm{m})$ objects. HD2 RGCs were better suited for $200 \mu \mathrm{m}$ objects, in part because their lack of complete surround suppression to positive contrast stimuli allows them to maintain higher firing rates, especially at high motion speeds. While UHD and HD2 RGCsboth nonobject-motion-sensitive RGC types (Fig. 9) — showed the smallest tracking error for 100 and $200 \mu \mathrm{m}$ objects, respectively, HD1 and LED RGCs-both object-motion-sensitive (Fig. 9)_-performed worse at the tracking task. Perhaps this suggests a distinction in the types of motion best represented by nonobjectmotion-sensitive RGC types, which can track small objects on a static background, and object-motion-sensitive RGCs, which compare local verses global motion. We only modeled the responses to moving ON edges, but since HD RGCs respond to both ON and OFF, they also encode the motion of OFF edges. Differences in ON/OFF ratio (Fig. 3) might also be important to downstream brain regions. Other perturbations of objectmotion stimuli would likely reveal additional specializations.

\section{Central projections and visual functions of $\mathrm{HD}$ and LED RGCs}

Despite previous studies characterizing the LED RGC, as well as an identified transgene that labels this cell in mice (Kim et al., 2010; Zhang et al., 2012), its projection pattern remains largely unknown. The primary reason for this may be that the W3 line does not label a single RGC type, making it difficult to isolate exactly where the brightly labeled "W3b" cells (LED RGCs) project specifically in relation to the other labeled cells (Kim et al., 2010). Overcoming this hurdle with more selective markers will provide future researchers tools to study the projection patterns of HD and LED RGCs to higher visual centers in the brain and will aid in assigning functional roles to these RGC types.

Classically, the dorsal lateral geniculate nucleus (dLGN) is considered a relay center that receives high-resolution visual information relevant to conscious visual perception; HD RGC projections to this region would imply that they are involved in relaying high-resolution vision. A recent investigation revealed that $(\mathrm{ON})$ RGCs with transient response properties and high sensitivity to small stimuli appear to innvervate the superior colliculus (SC), but not dLGN (Ellis et al., 2016). The SC is classically seen as a sensory motor integration center that guides aspects of vision, such as coordinated eye movements. It has also been implicated in controlling rapid, visually guided reflexive behaviors (Shang et al., 2015). Therefore, it is possible that the generally transient HD family of RGCs may indeed project to the SC, while the stereotypically sustained response properties of LED RGCs may serve a different purpose and perhaps project elsewhere.

Specific genetic markers for HD RGCs are currently unknown, but efforts to find RGC-specific markers are underway both in our laboratory and in those of others (Macosko et al., 2015). Trangenic lines selective for HD RGCs would enable not only tracing their axons into the brain but also in vivo ablation experiments to investigate their role in visually guided behavior. Even without the aid of HD RGC-specific mouse lines, retrograde tracing experiments from the dLGN, SC, and other retinorecipient areas, along with the functional targeting of HD RGCs described above, could implicate HD cells in particular facets of visual processing.

\section{References}

Asari H, Meister M (2012) Divergence of visual channels in the inner retina. Nat Neurosci 15:1581-1589. CrossRef Medline

Awatramani GB, Slaughter MM (2000) Origin of transient and sustained responses in ganglion cells of the retina. J Neurosci 20:7087-7095. Medline

Baccus SA, Olveczky BP, Manu M, Meister M (2008) A retinal circuit that computes object motion. J Neurosci 28:6807-6817. CrossRef Medline

Baden T, Berens P, Franke K, Román Rosón M, Bethge M, Euler T (2016) 
The functional diversity of retinal ganglion cells in the mouse. Nature 529:345-350. CrossRef Medline

Berry MJ 2nd, Brivanlou IH, Jordan TA, Meister M (1999) Anticipation of moving stimuli by the retina. Nature 398:334-338. CrossRef Medline

Bleckert A, Schwartz GW, Turner MH, Rieke F, Wong RO (2014) Visual space is represented by nonmatching topographies of distinct mouse retinal ganglion cell types. Curr Biol 24:310-315. CrossRef Medline

Borghuis BG, Marvin JS, Looger LL, Demb JB (2013) Two-photon imaging of nonlinear glutamate release dynamics at bipolar cell synapses in the mouse retina. J Neurosci 33:10972-10985. CrossRef Medline

Chen EY, Marre O, Fisher C, Schwartz G, Levy J, da Silviera RA, Berry MJ 2nd (2013) Alert response to motion onset in the retina. J Neurosci 33:120132. CrossRef Medline

Chen EY, Chou J, Park J, Schwartz G, Berry MJ 2nd (2014) The neural circuit mechanisms underlying the retinal response to motion reversal. J Neurosci 34:15557-15575. CrossRef Medline

Devries SH, Baylor DA (1997) Mosaic arrangement of ganglion cell receptive fields in rabbit retina. J Neurophysiol 78:2048-2060. Medline

Ellis EM, Gauvain G, Sivyer B, Murphy GJ (2016) Shared and distinct retinal input to the mouse superior colliculus and dorsal lateral geniculate nucleus. J Neurophysiol 116:602-610. CrossRef Medline

Farrow K, Masland RH (2011) Physiological clustering of visual channels in the mouse retina. J Neurophysiol 105:1516-1530. CrossRef Medline

Greene MJ, Kim JS, Seung HS, Seung HS (2016) Analogous convergence of sustained and transient inputs in parallel on and off pathways for retinal motion computation. Cell Rep 14:1892-1900. CrossRef Medline

Grimes WN (2012) Amacrine cell-mediated input to bipolar cells: variations on a common mechanistic theme. Vis Neurosci 29:41-49. CrossRef Medline

Helmstaedter M, Briggman KL, Turaga SC, Jain V, Seung HS, Denk W (2013) Connectomic reconstruction of the inner plexiform layer in the mouse retina. Nature 500:168-174. CrossRef Medline

Ishikane H, Gangi M, Honda S, Tachibana M (2005) Synchronized retinal oscillations encode essential information for escape behavior in frogs. Nat Neurosci 8:1087-1095. CrossRef Medline

Jacoby J, Zhu Y, DeVries SH, Schwartz GW (2015) An amacrine cell circuit for signaling steady illumination in the retina. Cell Rep 13:2663-2670. CrossRef Medline

Johnston J, Lagnado L (2015) General features of the retinal connectome determine the computation of motion anticipation. Elife 4:e06250. CrossRef Medline

Kim IJ, Zhang Y, Meister M, Sanes JR (2010) Laminar restriction of retinal ganglion cell dendrites and axons: subtype-specific development patterns revealed with transgenic markers. J Neurosci 30:1452-1462. CrossRef Medline

Kim JS, Greene MJ, Zlateski A, Lee K, Richardson M, Turaga SC, Purcaro M, Balkam M, Robinson A, Behabadi BF, Campos M, Denk W, Seung HS, Seung HS (2014) Space-time wiring specificity supports direction selectivity in the retina. Nature 509:331-336. CrossRef Medline

Kim T, Soto F, Kerschensteiner D (2015) An excitatory amacrine cell detects object motion and provides feature-selective input to ganglion cells in the mouse retina. Elife 4:6807. CrossRef Medline

Kolb H (1979) The inner plexiform layer in the retina of the cat: electron microscopic observations. J Neurocytol 8:295-329. CrossRef Medline

Krishnaswamy A, Yamagata M, Duan X, Hong YK, Sanes JR (2015) Sidekick 2 directs formation of a retinal circuit that detects differential motion. Nature 524:466-470. CrossRef Medline

Lee S, Chen L, Chen M, Ye M, Seal RP, Zhou ZJ (2014) An unconventional glutamatergic circuit in the retina formed by vGluT3 amacrine cells. Neuron 84:708-715. CrossRef Medline

Leonardo A, Meister M (2013) Nonlinear dynamics support a linear population code in a retinal target-tracking circuit. J Neurosci 33:16971-16982. CrossRef Medline

Lettvin JY, Maturana HR, McCulloch WS, Pitts WH (1959) What the frog's eye tells the frog's brain. Proc IRE 154:233-258. CrossRef

Levick WR (1967) Receptive fields and trigger features of ganglion cells in the visual streak of the rabbits retina. J Physiol 188:285-307. CrossRef Medline

Macosko EZ, Basu A, Satija R, Nemesh J, Shekhar K, Goldman M, Tirosh I, Bialas AR, Kamitaki N, Martersteck EM, Trombetta JJ, Weitz DA, Sanes JR, Shalek AK, Regev A, McCarroll SA (2015) Highly parallel genome- wide expression profiling of individual cells using nanoliter droplets. Cell 161:1202-1214. CrossRef Medline

Masland RH (2001) The fundamental plan of the retina. Nat Neurosci 4:877-886. CrossRef Medline

Masland RH (2012a) The tasks of amacrine cells. Vis Neurosci 29:3-9. CrossRef Medline

Masland RH (2012b) The neuronal organization of the retina. Neuron 76: 266-280. CrossRef Medline

Nath A, Schwartz GW (2016) Cardinal orientation selectivity is represented by two distinct ganglion cell types in mouse retina. J Neurosci 36:32083221. CrossRef Medline

Olveczky BP, Baccus SA, Meister M (2003) Segregation of object and background motion in the retina. Nature 423:401-408. CrossRef Medline

Pearson JT, Kerschensteiner D (2015) Ambient illumination switches contrast preference of specific retinal processing streams. J Neurophysiol 114:540-550. CrossRef Medline

Peichl L, Wässle H (1983) The structural correlate of the receptive field centre of alpha ganglion cells in the cat retina. J Physiol 341:309-324. CrossRef Medline

Rousso DL, Qiao M, Kagan RD, Yamagata M, Palmiter RD, Sanes JR (2016) Two pairs of ON and OFF retinal ganglion cells are defined by intersectional patterns of transcription factor expression. Cell Rep 15:1930-1944. CrossRef Medline

Sanes JR, Masland RH (2015) The types of retinal ganglion cells: current status and implications for neuronal classification. Annu Rev Neurosci 38:221-246. CrossRef Medline

Schwartz G, Harris R, Shrom D, Berry MJ 2nd (2007) Detection and prediction of periodic patterns by the retina. Nat Neurosci 10:552-554. CrossRef Medline

Schwartz GW, Okawa H, Dunn FA, Morgan JL, Kerschensteiner D, Wong RO, Rieke F (2012) The spatial structure of a nonlinear receptive field. Nat Neurosci 15:1572-1580. CrossRef Medline

Semmelhack JL, Donovan JC, Thiele TR, Kuehn E, Laurell E, Baier H (2014) A dedicated visual pathway for prey detection in larval zebrafish. Elife 3:17968. CrossRef Medline

Shang C, Liu Z, Chen Z, Shi Y, Wang Q, Liu S, Li D, Cao P (2015) Brain circuits. A parvalbumin-positive excitatory visual pathway to trigger fear responses in mice. Science 348:1472-1477. CrossRef Medline

Sümbül U, Song S, McCulloch K, Becker M, Lin B, Sanes JR, Masland RH, Seung HS (2014) A genetic and computational approach to structurally classify neuronal types. Nat Commun 5:3512. CrossRef Medline

Takeshita D, Gollisch T (2014) Nonlinear spatial integration in the receptive field surround of retinal ganglion cells. J Neurosci 34:75487561. CrossRef Medline

Temizer I, Donovan JC, Baier H, Semmelhack JL (2015) A visual pathway for looming-evoked escape in larval zebrafish. Curr Biol 25:1823-1834. CrossRef Medline

Thoreson WB, Mangel SC (2012) Lateral interactions in the outer retina. Prog Retin Eye Res 31:407-441. CrossRef Medline

Tikidji-Hamburyan A, Reinhard K, Seitter H, Hovhannisyan A, Procyk CA, Allen AE, Schenk M, Lucas RJ, Münch TA (2015) Retinal output changes qualitatively with every change in ambient illuminance. Nat Neurosci 18:66-74. CrossRef Medline

Trenholm S, Johnson K, Li X, Smith RG, Awatramani GB (2011) Parallel mechanisms encode direction in the retina. Neuron 71:683-694. CrossRef Medline

Trenholm S, McLaughlin AJ, Schwab DJ, Awatramani GB (2013) Dynamic tuning of electrical and chemical synaptic transmission in a network of motion coding retinal neurons. J Neurosci 33:14927-14938. CrossRef Medline

Wässle H, Boycott BB (1991) Functional architecture of the mammalian retina. Physiol Rev 71:447-480. Medline

WässleH, Peichl L, Boycott BB (1981) Dendritic territories of cat retinal ganglion cells. Nature 292:344-345. CrossRef Medline

Yang G, Masland RH (1994) Receptive fields and dendritic structure of directionally selective retinal ganglion cells. J Neurosci 14:5267-5280. Medline

Yilmaz M, Meister M (2013) Rapid innate defensive responses of mice to looming visual stimuli. Curr Biol 23:2011-2015. CrossRef Medline

Zhang Y, Kim IJ, Sanes JR, Meister M (2012) The most numerous ganglion cell type of the mouse retina is a selective feature detector. Proc Natl Acad Sci U S A 109:E2391-E2398. CrossRef Medline 